\title{
Efficient glueball simulations on anisotropic lattices
}

\author{
Colin J. Morningstar \\ Department of Physics, University of California at San Diego, La Jolla, California 92093-0319 \\ Mike Peardon \\ Department of Physics and Astronomy, University of Kentucky, Lexington, Kentucky 40506-0055
}

(Received 23 April 1997)

\begin{abstract}
Monte Carlo results for the low-lying glueball spectrum using an improved, anisotropic action are presented. Ten simulations at lattice spacings ranging from 0.2 to $0.4 \mathrm{fm}$ and two different anisotropies have been performed in order to demonstrate the advantages of using coarse, anisotropic lattices to calculate glueball masses. Our determinations of the tensor $\left(2^{++}\right)$and pseudovector $\left(1^{+-}\right)$glueball masses are more accurate than previous Wilson action calculations. [S0556-2821(97)00419-0]

PACS number(s): $12.38 . \mathrm{Gc}, 11.15 . \mathrm{Ha}, 12.39 . \mathrm{Mk}$
\end{abstract}

\section{INTRODUCTION}

Numerical simulations of gluons on a space-time lattice provide at present the most reliable means of studying glueballs. Glueball correlation functions are, however, notoriously difficult quantities to measure in Monte Carlo simulations: since the masses of these states are rather high and their creation operators have large vacuum fluctuations, the signal-to-noise ratio falls extremely rapidly as the separation between the source and sink is increased. Because of this, reliable studies of glueballs on fine lattices have required prohibitively large computer resources. Thus, the development of more efficient simulation techniques in lattice QCD is crucial to establishing a detailed description of glueballs and their interactions.

The objective of this work is to examine the effectiveness of using an improved, anisotropic lattice action to reduce the computational effort needed to determine the glueball spectrum in quenched QCD. Improved actions allow access to continuum physics on coarser lattices than possible using the simple Wilson discretization. Coarse lattice simulations are more efficient for several reasons: for a given physical volume, much fewer lattice sites are needed; the alleviation of critical slowing down permits the faster generation of statistically independent gauge-field configurations; glueball operator smearing is faster due to the decreased number of links and a decrease in the number of smearing iterations required; glueball wave functions extend over much fewer lattice sites on a coarse lattice, making the variational technique far more effective when using a feasible number (a dozen or so) of basis operators.

However, for glueball mass calculations, the coarseness of the temporal lattice spacing is a severe drawback. As the masses in lattice units of the states of interest are so large, the number of correlator time intervals which can be measured is reduced greatly [1]. A straightforward solution to this problem which preserves the computational advantages of coarse lattices [2-4] is to make use of anisotropic lattices in which the temporal spacing is much smaller than that in the spatial directions. This enables us to exploit the enhanced signal-to-noise ratio of the correlation functions at smaller temporal separations. A natural scale for the temporal lattice spacing should be the inverse of the energy of the states of interest; thus, for glueballs, a temporal cutoff larger than $1.5 \mathrm{GeV}$ allows resolution from accessible statistics of the correlator over a few time slices. Meanwhile, the scale for the spatial lattice should be set by the size of the wave function of the state; a spatial grid separation in the range $0.2-0.4$ fm would seem reasonable.

Since we propose to use lattices in which the temporal lattice spacing is small, improvement of the discretization in this direction is not needed. Thus, a lattice action which couples only nearest-neighbor time slices can be used. The transfer matrix corresponding to such an action is Hermitian and positive definite; all of our effective masses must converge to their plateau values monotonically from above. This ensures the validity of variational techniques which minimize the effective masses at small temporal separations. Such techniques are very effective in diminishing the excited-state contributions to the glueball correlation functions and are crucial for efficient extraction of ground-state masses.

In this paper, we demonstrate the increased efficiency of glueball simulations using these actions on anisotropic lattices. We present results for the masses of three of the lighter SU(3) glueball states, the scalar $\left(0^{++}\right)$, the tensor $\left(2^{++}\right)$, and the pseudovector $\left(1^{+-}\right)$. The masses of the first-excited states in the scalar and tensor channels were also examined. Ten simulations at lattice spacings ranging from 0.2 to 0.4 fm were performed, enabling reliable extrapolations to the continuum limit (although the mass of the scalar glueball was somewhat problematic). The results are compared to previous simulation data obtained using the Wilson action and we find that more accurate determinations of the tensor and pseudovector glueball masses have been achieved. A comparison of efficiencies is also made. Lastly, finitevolume effects are shown to be small.

The new action used in our simulations is described in Sec. II. The details of the glueball simulations, including the construction of the glueball operators, the generation of the gauge-field configurations, and the analysis of the Monte Carlo data, are given in Sec. III. The hadronic scale $r_{0}$ is used to relate our results at different values of the coupling $\beta$ and the aspect ratio $\xi$. The determination of this scale in 
terms of the lattice spacing using the static potential is outlined in Sec. IV. Section V contains our results and discussion: the glueball mass measurements are presented in detail; finite-volume effects are studied; extrapolations of the masses at finite spacing to the continuum limit are undertaken; the conversion of our results into physical units is described; and a comparison of efficiencies with Wilson action simulations is made. Our conclusions are given in Sec. VI, along with an outline of future work.

\section{AN IMPROVED, ANISOTROPIC DISCRETIZATION OF QCD}

Our glueball mass determinations rely on numerical simulations of glueballs on a Euclidean space-time lattice with spatial and temporal spacings $a_{s}$ and $a_{t}$, respectively. The improved gluonic action used in this study is given by $[2,4]$

$$
S_{I I}=\beta\left\{\frac{5}{3} \frac{\Omega_{\mathrm{sp}}}{\xi u_{s}^{4}}+\frac{4}{3} \frac{\xi \Omega_{\mathrm{tp}}}{u_{s}^{2} u_{t}^{2}}-\frac{1}{12} \frac{\Omega_{\mathrm{sr}}}{\xi u_{s}^{6}}-\frac{1}{12} \frac{\xi \Omega_{\mathrm{str}}}{u_{s}^{4} u_{t}^{2}}\right\},
$$

where $\beta=6 / g^{2}, g$ is the QCD coupling, $u_{s}$ and $u_{t}$ are the mean-link renormalization parameters, $\xi$ is the aspect ratio $\left(\xi=a_{s} / a_{t}\right.$ at the tree level in perturbation theory), and $\Omega_{C}=\Sigma_{C} \frac{1}{3} \operatorname{Re} \operatorname{Tr}\left(1-W_{C}\right)$, with $W_{C}$ denoting the pathordered product of link variables along a closed contour $C$ on the lattice. $\Omega_{\mathrm{sp}}$ includes the sum over all spatial plaquettes on the lattice, $\Omega_{\text {tp }}$ indicates the temporal plaquettes, $\Omega_{\text {sr }}$ denotes the product of link variables about planar $2 \times 1$ spatial rectangular loops, and $\Omega_{\text {str }}$ refers to the short temporal rectangles (one temporal link, two spatial). Explicitly,

$$
\Omega_{\mathrm{sp}}=\sum_{x} \sum_{i>j} \frac{1}{3} \operatorname{Re} \operatorname{Tr}\left[1-U_{i}(x) U_{j}(x+\hat{\imath}) U_{i}^{\dagger}(x+\hat{\jmath}) U_{j}^{\dagger}(x)\right],
$$

$$
\Omega_{\mathrm{tp}}=\sum_{x} \sum_{i} \frac{1}{3} \operatorname{Re} \operatorname{Tr}\left[1-U_{t}(x) U_{i}(x+\hat{t}) U_{t}^{\dagger}(x+\hat{\imath}) U_{i}^{\dagger}(x)\right],
$$

$$
\begin{aligned}
\Omega_{\mathrm{sr}}= & \sum_{x} \sum_{i \neq j} \frac{1}{3} \operatorname{Re} \operatorname{Tr}\left[1-U_{i}(x) U_{i}(x+\hat{\imath}) U_{j}(x+2 \hat{\imath})\right. \\
& \left.\times U_{i}^{\dagger}(x+\hat{\imath}+\hat{\jmath}) U_{i}^{\dagger}(x+\hat{\jmath}) U_{j}^{\dagger}(x)\right], \\
\Omega_{\mathrm{str}}= & \sum_{x} \sum_{i} \frac{1}{3} \operatorname{Re} \operatorname{Tr}\left[1-U_{i}(x) U_{i}(x+\hat{\imath}) U_{t}(x+2 \hat{\imath})\right. \\
& \left.\times U_{i}^{\dagger}(x+\hat{\imath}+\hat{t}) U_{i}^{\dagger}(x+\hat{t}) U_{t}^{\dagger}(x)\right],
\end{aligned}
$$

where $x$ labels the sites of the lattice, $i, j$ are spatial indices, and $U_{\mu}(x)$ is the parallel transport matrix in the gluon field from site $x$ to $x+\hat{\mu}$.

This action, intended for use with $a_{t} \ll a_{s}$, has $O\left(a_{s}^{4}, a_{t}^{2}, \alpha_{s} a_{s}^{2}\right)$ discretization errors. The $O\left(a_{t}^{2}\right)$ errors can be removed by the addition of counterterms which couple next-nearest-neighbor time slices, but this introduces spurious high-energy modes which can cause considerable problems for our glueball simulations. These unphysical states appear in perturbation theory as additional poles in the gluon propagator. Their detrimental effects on the glueball correla- tion functions have been previously demonstrated [1]. Although these spurious states do not affect the asymptotic behavior of the glueball correlators, they do appreciably change the correlators at short temporal separations and can seriously hinder attempts to reduce excited-state contamination to hasten the onset of asymptotic behavior. Since our glueball mass measurements rely heavily on the reduction of such excited-state contributions to the glueball correlation functions, the use of an action which is free of spurious lattice modes is crucial. The action given in Eq. (1) couples only link variables on neighboring time slices, which ensures that all of our effective masses converge to their plateau values monotonically from above and so validates the variational techniques employed.

It is now known that perturbation theory by itself does not reliably determine the couplings in an improved action in lattice gauge theory. Hence, the interaction strengths in $S_{I I}$ have been determined using a judicious combination of perturbation theory and mean-field theory. Mean-field theory is introduced by separately renormalizing the spatial and temporal link variables: $U_{j}(x) \rightarrow U_{j}(x) / u_{s}$ and $U_{t}(x) \rightarrow$ $U_{t}(x) / u_{t}$, where $u_{s}$ and $u_{t}$ denote the renormalization factors for the spatial and temporal links, respectively. The mean-link parameters $u_{t}$ and $u_{s}$ are best determined by guessing input values for use in the action, measuring the mean links in the Landau gauge in a simulation, then readjusting the input values accordingly and tuning until the input values match the measured values. The determination of these renormalization factors is described in more detail in Refs. [2,4]. However, when $a_{t}$ is significantly smaller than $a_{s}$, we expect the mean temporal link $u_{t}$ to be very close to unity since $1-\left\langle\frac{1}{3} \operatorname{Tr} U_{t}\right\rangle \propto\left(a_{t} / a_{s}\right)^{2}$ in perturbation theory. Hence, to simplify matters, we set $u_{t}=1$. We introduce further simplifications by using a convenient and gaugeinvariant definition for $u_{s}$ in terms of the mean spatial plaquette given by $u_{s}=\left\langle\frac{1}{3} \operatorname{Re} \operatorname{Tr} P_{s s^{\prime}}\right\rangle^{1 / 4}$, where $P_{s s^{\prime}}$ denotes the spatial plaquette. This eliminates the need for gauge fixing, yields values for $u_{s}$ which differ from those found using the Landau gauge definition by only a few percent, and significantly speeds up the tuning process.

At finite coupling $g$, the anisotropy $a_{s} / a_{t}$ is renormalized away from its input value $\xi$. Measurements of this renormalization have been made using the static potential extracted from correlations along the different spatial and temporal axes of the lattice $[2,4]$. Without mean-link improvement, this renormalization can be as large as $30 \%$. When the action includes mean-link corrections, this renormalization is found to be small, typically a few percent. We used $a_{t} / a_{s}=\xi$ in all of our calculations, accepting the small radiative corrections to the anisotropy as finite lattice spacing errors, which vanish in the continuum limit.

\section{GLUEBALL SIMULATION DETAILS}

Glueballs may be labeled by their total (integral) spin $J$ and their symmetries under spatial inversion and charge conjugation. However, on a cubic lattice, glueballs are characterized by their transformation properties under the cubic point group, combined with parity and charge conjugation operations. The cubic group $O_{h}$ has 24 elements that fall into 
five conjugacy classes, and thus, the dimensions of the irreducible representations (irreps) are 1, 1, 2, 3, and 3. These irreps are labeled $A_{1}, A_{2}, E, T_{1}$, and $T_{2}$, respectively. Including parity and charge conjugation symmetry operations, there are 20 irreps (labeled by $J^{P C}$, where $J$ now denotes an irrep of $O_{h}$ ). In this study, four of the irreps which generate light $(<3 \mathrm{GeV})$ glueball states were simulated: the $A_{1}^{++}$, $E^{++}, T_{2}^{++}$, and $T_{1}^{+-}$channels. Of particular interest are the $E^{++}$and $T_{2}^{++}$irreps whose combined five rows correspond to the five polarization states of the tensor $\left(2^{++}\right)$glueball which become degenerate as continuum rotational invariance is restored. This then gives information on the magnitude of lattice artifacts at finite cutoff.

The mass of a glueball $G$ having a given $J^{P C}$ can be extracted from the large- $t$ behavior of a correlation function $C(t)=\left\langle 0\left|\bar{\Phi}^{(R) \dagger}(t) \bar{\Phi}^{(R)}(0)\right| 0\right\rangle$, where $R$ denotes the lattice irrep corresponding to the $J^{P C}$ of interest and $\bar{\Phi}^{(R)}(t)=\Phi^{(R)}(t)-\left\langle 0\left|\Phi^{(R)}(t)\right| 0\right\rangle$ is a gauge-invariant, translationally invariant, vacuum-subtracted operator capable of creating a glueball out of the QCD vacuum $|0\rangle$. As the temporal separation $t$ becomes large, this correlator tends to a single decaying exponential $\lim _{t \rightarrow \infty} C(t)=Z \exp \left(-m_{G} t\right)$, where $m_{G}$ is the mass of the lowest-lying glueball which can be created by the operator $\bar{\Phi}^{(R)}(t)$. In order to extract $m_{G}$, the correlator $C(t)$ must be determined for $t$ sufficiently large that $C(t)$ is well approximated by its asymptotic form. However, the signal-to-noise ratio in any Monte Carlo determination of $C(t)$ falls exponentially fast with respect to $t$. Thus, it is crucial to use a glueball operator for which $C(t)$ attains its asymptotic form as quickly as possible. If $|G\rangle$ denotes the glueball state of interest, this means that we must choose an operator for which the overlap $\left\langle G\left|\bar{\Phi}^{(R)}(t)\right| 0\right\rangle /\left[\langle G \mid G\rangle\left\langle 0\left|\bar{\Phi}^{(R) \dagger}(t) \bar{\Phi}^{(R)}(t)\right| 0\right\rangle\right]^{1 / 2}$ is as near to unity as possible. For such an operator, the signal-to-noise ratio is also optimal [5].

In order to construct such operators, we exploited the smearing [6,7] and variational techniques which have been used with success in earlier Wilson action simulations. In each of the $J^{P C}$ channels of interest, glueball operators were constructed on each time slice in a sequence of three steps. First, smeared links $U_{j}^{s}(x)$ and fuzzy superlinks $U_{j}^{f}(x)$ were formed. Second, a set of basic operators $\phi_{\alpha}^{(R)}(t)$ were constructed using linear combinations of gauge-invariant, pathordered products of the $U_{j}^{s}(x)$ and $U_{j}^{f}(x)$ matrices about various closed spatial loops; each such linear combination was designed to be invariant under spatial translations and to transform irreducibly under the symmetry operations of the cubic point group according to the irrep of interest. Last, the glueball operators $\Phi^{(R)}(t)$ were formed from linear combinations of the basic operators, $\Phi^{(R)}(t)=\Sigma_{\alpha} v_{\alpha}^{(R)} \phi_{\alpha}^{(R)}(t)$, where the coefficients $v_{\alpha}^{(R)}$ were determined using the variational method. Each of these three steps is described below.

Operators constructed out of smeared links and fuzzy superlinks have dramatically reduced mixings with the high frequency modes of the theory. Thus, the use of spatially smoothed links is an important part of reducing excited-state contamination in the glueball correlation functions. Two smoothening procedures were used: a single-link procedure and a double-link procedure. In the single-link procedure, every spatial link $U_{j}(x)$ on the lattice is replaced by itself plus a sum of its four neighboring (spatial) staples, projected back into $\mathrm{SU}(3)$ :

$$
\begin{aligned}
U_{j}^{s}(x)= & \mathcal{P}_{\mathrm{SU}(3)}\left\{U_{j}(x)+\lambda_{s} \sum_{ \pm(k \neq j)}\right. \\
& \left.\times U_{k}(x) U_{j}(x+\hat{k}) U_{k}^{\dagger}(x+\hat{\jmath})\right\},
\end{aligned}
$$

where $\mathcal{P}_{\mathrm{SU}(3)}$ denotes the projection [8] into SU(3). Here, we denote this mapping of the spatial link matrices into the smeared link variables by $s_{\lambda_{s}}$. In the double-link procedure, new superlinks $U^{f}$ of length $2 a_{s}$ are built using neighboring staples which connect sites separated by a distance twice the length of the source link variables:

$$
\begin{aligned}
U_{j}^{f}(x)= & \mathcal{P}_{\mathrm{SU}(3)}\left\{U_{j}(x) U_{j}(x+\hat{\jmath})+\lambda_{f} \sum_{ \pm(k \neq j)} U_{k}(x)\right. \\
& \left.\times U_{j}(x+\hat{k}) U_{j}(x+\hat{\jmath}+\hat{k}) U_{k}^{\dagger}(x+2 \hat{\jmath})\right\},
\end{aligned}
$$

and we denote this mapping by $f_{\lambda_{f}}$. Both procedures can be applied recursively; smeared links can be smeared again and fuzzy links of increasing length $2 a_{s}, 4 a_{s}, 8 a_{s}, \ldots$ can be constructed. A smoothening scheme $\mathcal{S}$ is defined as a composition of single-link mappings and double-link mappings. Six different smoothening schemes were used. The simplest scheme used was the composition of two single-link smearings: $\mathcal{S}_{1}=s_{\lambda_{s}}{ }^{\circ} s_{\lambda_{s}}$. To simplify notation, we write this as $\mathcal{S}_{1}=s_{\lambda_{s}}^{2}$. We also used the compositions of four and six single-link mappings: $\mathcal{S}_{2}=s_{\lambda_{s}}^{4}$ and $\mathcal{S}_{3}=s_{\lambda_{s}}^{6}$. In the other three smoothening schemes, the application of several single-link smearings, followed by one final iteration of double-link fuzzing was used: $\mathcal{S}_{4}=f_{\lambda_{f}}{ }^{\circ} s_{\lambda_{s}}^{2}, \quad \mathcal{S}_{5}=f_{\lambda_{f}}{ }^{\circ} s_{\lambda_{s}}^{4}$, and $\mathcal{S}_{6}$ $=f_{\lambda_{f}}{ }^{\circ} s_{\lambda_{s}}^{6}$. Only one iteration of the fuzzing procedure which results in links connecting sites separated by $2 a_{s}$ was found to be useful for the range of coarse $a_{s}$ values explored here. For the finer lattices $(\beta=2.4, \xi=5$, and $\beta=2.6, \xi=3)$, an extra four initial iterations of single-link smearing were used in all six smoothening schemes to enhance ground-state overlap. To simplify matters, the same values for the two parameters $\lambda_{s}$ and $\lambda_{f}$ were used in all smearing and fuzzing iterations. These values were chosen to minimize excitedstate contamination in the glueball correlation functions. A crude optimization was done in a set of low statistics runs and the optimal values $\lambda_{s}=0.1$ and $\lambda_{f}=0.5$ were then used in all the glueball simulations.

The second step in the construction of our glueball operators was the formation of a set of basic operators $\phi_{\alpha}^{(R)}(t)$ using linear combinations of gauge-invariant, path-ordered products of the $U_{j}^{s}(x)$ and $U_{j}^{f}(x)$ matrices about various closed spatial loops. Combinations which were Hermitian, invariant under spatial translations, and transformed irreducibly under the operations of the cubic point group according to the irrep of interest were constructed. For a more detailed exposition of this construction, see Ref. [9]. In each channel, a large set of prototypes was programmed, and a short simu- 
$A_{1}^{++}$
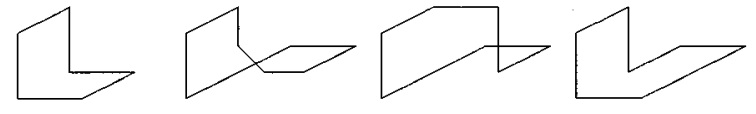

$E^{++}$
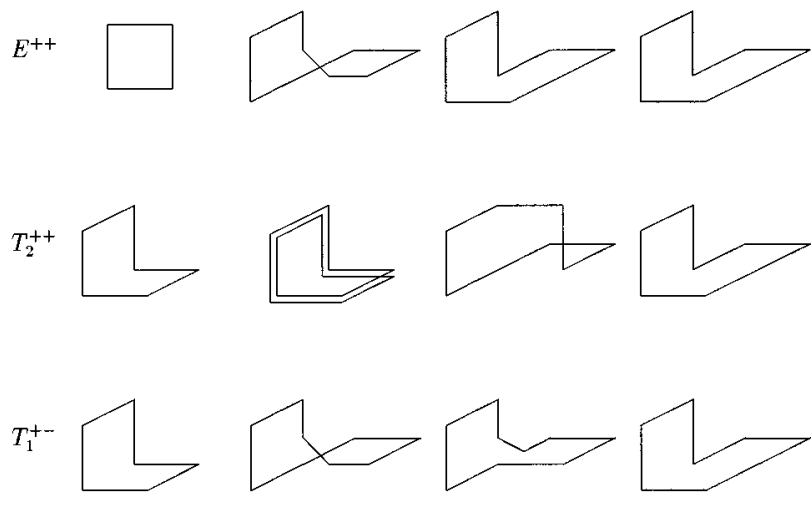

FIG. 1. The four Wilson loop shapes in each channel used to form the lattice glueball operators. The complete set of 24 operators was formed by computing linear combinations of each of these loops rotated and translated across the lattice on six different sets of smoothed links. Where a loop shape occurs twice, it is used in two different projections into the appropriate irreducible representation.

lation was then performed to determine the coefficients of each operator in the variational ground state. In each channel, the four operators with the highest of these contributions were then chosen for use in the production runs. The paths in this optimal set are illustrated in Fig. 1. In the glueball simulations, these Wilson loops were measured on the link variables from the six smoothening schemes, yielding a total of $N=24$ basic operators $\phi_{\alpha}^{(R)}(t)$ in each of the four channels.

Finally, $\Phi^{(R)}(t)$ was formed from a linear combination of the basic operators, $\Phi^{(R)}(t)=\sum_{\alpha=1}^{N} v_{\alpha}^{(R)} \phi_{\alpha}^{(R)}(t)$. The coefficients $v_{\alpha}^{(R)}$ were determined using the variational method. First, the $24 \times 24$ correlation matrix was computed in the glueball simulations:

$$
\widetilde{C}_{\alpha \beta}(t)=\sum_{\tau}\left\langle 0\left|\bar{\phi}_{\alpha}^{(R)}(\tau+t) \bar{\phi}_{\beta}^{(R)}(\tau)\right| 0\right\rangle,
$$

where $\bar{\phi}_{\alpha}^{(R)}(t)$ denotes a vacuum-subtracted operator $\bar{\phi}_{\alpha}^{(R)}(t)=\phi_{\alpha}^{(R)}(t)-\left\langle 0\left|\phi_{\alpha}^{(R)}(t)\right| 0\right\rangle$. Note that $\left\langle 0\left|\phi_{\alpha}^{(R)}(t)\right| 0\right\rangle$ is independent of $t$. The coefficients $v_{\alpha}^{(R)}$ were then determined by minimizing the effective mass

$$
\widetilde{m}\left(t_{D}\right)=-\ln \left[\frac{\sum_{\alpha \beta} v_{\alpha}^{(R)} v_{\beta}^{(R)} \widetilde{C}_{\alpha \beta}\left(t_{D}\right)}{\sum_{\alpha \beta} v_{\alpha}^{(R)} v_{\beta}^{(R)} \widetilde{C}_{\alpha \beta}(0)}\right],
$$

where the time separation for optimization was fixed in all cases to $t_{D}=1$. Let $\mathbf{v}^{(R)}$ denote a column vector whose elements are the optimal values of the coefficients $v_{\alpha}^{(R)}$. Then requiring $d \widetilde{m}\left(t_{D}\right) / d v_{\alpha}^{(R)}=0$ for all $\alpha$ yields an eigenvalue equation:

$$
\widetilde{C}\left(t_{D}\right) \mathbf{v}^{(R)}=e^{-\widetilde{m}\left(t_{D}\right)} \widetilde{C}(0) \mathbf{v}^{(R)} .
$$

TABLE I. Input parameters used in the glueball simulations.

\begin{tabular}{llll}
\hline \hline$\beta$ & $\xi$ & Lattice & \multicolumn{1}{c}{$u_{s}^{4}$} \\
\hline 1.7 & 3 & $6^{3} \times 18$ & 0.3075 \\
1.9 & 3 & $6^{3} \times 18$ & 0.340 \\
2.0 & 3 & $8^{3} \times 24$ & 0.356 \\
2.2 & 3 & $8^{3} \times 24$ & 0.3885 \\
2.4 & 3 & $8^{3} \times 24$ & 0.421 \\
2.6 & 3 & $10^{3} \times 30$ & 0.4505 \\
1.7 & 5 & $6^{3} \times 30$ & 0.295 \\
1.9 & 5 & $6^{3} \times 30$ & 0.328 \\
2.2 & 5 & $8^{3} \times 40$ & 0.378 \\
2.4 & 5 & $8^{3} \times 40$ & 0.409 \\
\hline \hline
\end{tabular}

The eigenvector $\mathbf{v}_{0}^{(R)}$ corresponding to the largest eigenvalue $e^{-\widetilde{m}_{0}\left(t_{D}\right)}$ then yields the coefficients $v_{0 \alpha}^{(R)}$ for the operator $\Phi_{0}^{(R)}(t)$ which best overlaps the lowest-lying glueball $G$ in the channel of interest. Operators which overlap excited glueball states can also be constructed using the other eigenvectors of Eq. (10). In particular, the operator $\Phi_{1}^{(R)}(t)$ expected to best overlap the first-excited glueball state $G^{*}$ was obtained from the eigenvector corresponding to the second largest eigenvalue of Eq. (10).

The elements of the correlator matrix given in Eq. (8) were estimated using the Monte Carlo method. Ten separate glueball simulations were performed on DEC Alpha workstations. Configuration ensembles were generated using both Cabibbo-Marinari (CM) pseudo-heat-bath and SU(2) subgroup over-relaxation (OR) methods. Link variables were updated in serial order on the lattice. We define a compound sweep as one CM updating sweep followed by three OR sweeps. In the glueball simulations, three compound sweeps were performed between measurements, and the measurements were averaged into bins of 100 in order to reduce data storage requirements (except for the $\beta=2.6, \xi=3$ run in which 40 configurations were included in each bin). In all ten simulations, 100 bins were obtained. Our ensembles were tested for residual autocorrelations during the analysis phase by overbinning by factors of 2 and 4 ; in all cases, the statistical error estimates remained unchanged.

Values for the mean-link parameter $u_{s}$ were determined self-consistently as previously described. This tuning procedure required a minimal amount of computational effort and provided thermalized configurations for later computations. The improved action simulation parameters used are given in Table I.

For the data-fitting phase, the large $24 \times 24$ correlator matrices in each channel were reduced using the coefficients $\mathbf{v}_{0}^{(R)}$ and $\mathbf{v}_{1}^{(R)}$ to smaller $2 \times 2$ matrices $C_{A B}(t)$ for $A, B=0,1$ :

$$
C_{A B}(t)=\sum_{\tau}\left\langle 0\left|\bar{\Phi}_{A}^{(R)}(\tau+t) \bar{\Phi}_{B}^{(R)}(\tau)\right| 0\right\rangle .
$$

The ground-state correlator $C_{00}(t)$ was fit for $t=t_{\min }, \ldots, t_{\max }$ using a single exponential

$$
C_{00}(t)=Z_{00}\left\{e^{-m_{G} t}+e^{-m_{G}(T-t)}\right\},
$$


TABLE II. Various run parameters for the static-quark potential measurements, including lattice sizes, total numbers of configurations used, and parameters for the two different smearing schemes.

\begin{tabular}{llcccc}
\hline \hline$\beta$ & $\xi$ & Lattice & No. configs. & $\left(\lambda_{1}, n_{\lambda 1}\right)$ & $\left(\lambda_{2}, n_{\lambda 2}\right)$ \\
\hline 1.7 & 3 & $6^{3} \times 18$ & 2275 & $(0.05,4)$ & $(0.10,4)$ \\
1.9 & 3 & $6^{3} \times 18$ & 1280 & $(0.07,4)$ & $(0.14,4)$ \\
2.0 & 3 & $8^{3} \times 24$ & 848 & $(0.07,4)$ & $(0.15,4)$ \\
2.2 & 3 & $8^{3} \times 24$ & 1024 & $(0.10,6)$ & $(0.16,6)$ \\
2.4 & 3 & $8^{3} \times 24$ & 1024 & $(0.12,6)$ & $(0.25,6)$ \\
2.6 & 3 & $10^{3} \times 30$ & 1100 & $(0.25,6)$ & $(0.50,6)$ \\
1.7 & 5 & $8^{3} \times 40$ & 810 & $(0.10,6)$ & $(0.25,6)$ \\
1.9 & 5 & $6^{3} \times 30$ & 1024 & $(0.08,4)$ & $(0.16,4)$ \\
2.2 & 5 & $12^{3} \times 48$ & 315 & $(0.20,4)$ & $(0.30,4)$ \\
2.4 & 5 & $8^{3} \times 40$ & 548 & $(0.20,6)$ & $(0.40,6)$ \\
\hline \hline
\end{tabular}

where $T$ was the temporal extent of the periodic lattice, to obtain an estimate of the mass $m_{G}$ (in terms of $a_{t}^{-1}$ ) of the lowest-lying glueball in each channel. To determine the mass $m_{G^{*}}$ of the first-excited glueball and another estimate of $m_{G}$, the $2 \times 2$ correlator was also fit for $t=t_{\min }, \ldots, t_{\max }$ using the form

$$
C_{A B}(t)=\sum_{p=G, G^{*}} Z_{A p} Z_{B p}\left\{e^{-m_{p} t}+e^{-m_{p}(T-t)}\right\} .
$$

Various fit regions $t_{\min }$ to $t_{\max }$ were used in order to check for consistency in the extracted values for the masses. Bestfit values were obtained using the correlated $\chi^{2}$ method. Error estimates were calculated using a 1024-point bootstrap procedure; in all cases, error estimates were very close to being symmetric about the central best-fit values and were thus averaged to simplify presentation.

\section{SETTING THE SCALE USING THE STATIC POTENTIAL}

In order to convert the glueball masses as measured in our simulations into physical units, we must set the scale by determining the lattice spacing $a_{t}$ for each $\beta$ and $\xi$ we consider. To do this, we must first choose one physical quantity to use as a reference. This reference quantity must then be measured on the lattice in terms of $a_{t}$. The experimentally known value for the reference quantity is then used to extract the lattice spacing. A quantity which can be easily and accurately determined both experimentally and in numerical simulations is an ideal choice for such a reference. The mass of a low-lying particle is typically used for setting the scale. In our case, however, there are no unambiguous experimental determinations of the glueball masses, so instead, we must look for another purely gluonic quantity.

The hadronic scale parameter $r_{0}$ defined in terms of the force between static quarks by $\left[r^{2} d V(\vec{r}) / d r\right]_{r=r_{0}}=1.65$, where $V(\vec{r})$ is the static-quark potential, is an attractive possibility. It can be measured very accurately on the lattice. The advantages in using $r_{0}$ to set the scale have been enumerated in Ref. [10]. From phenomenological potential models, one finds $r_{0} \approx 0.5 \mathrm{fm}$. A disadvantage in using $r_{0}$ is that its physical value must be deduced indirectly from experiment, and there is some ambiguity in doing this, as will be discussed below. However, in the absence of a better gluonic reference, we have chosen $r_{0}$ to set the scale. In this section, we outline the determination of $r_{0}$ in terms of $a_{s}$.

In order to determine $r_{0}$ in terms of the lattice spacing, we need accurate measurements of the static-quark potential. We extracted $V(\vec{r})$ for various spatial separations $\vec{r}$, both on and off the axes of the lattice, from the expectation values of Wilson loops $W(\vec{r}, t)$ in the standard manner:

$$
W(\vec{r}, t)=Z(\vec{r}) \exp [-t V(\vec{r})]+\text { excited-state contributions. }
$$

In the Monte Carlo evaluation of the Wilson loops, measurements were taken after every four compound sweeps (as defined in Sec. III). The measurements of the Wilson loops were done independently of the glueball mass studies using separate ensembles of configurations. To minimize contamination from excited states, the Wilson loops were constructed from iteratively smeared spatial links. The singlelink smearing method described previously was used. A given smearing scheme is specified not only by the parameter $\lambda_{s}$, but also by the total number of smearing iterations, denoted by $n_{\lambda}$. Two different choices of the smearing parameter were used in all cases: one smearing was chosen to work well for small $r=|\vec{r}|$, the other to work well for large $r$. Separate measurements for each smearing were taken; cross correlations were not determined. The statistical noise in the evaluation of $W(\vec{r}, t)$ was reduced dramatically, especially for large temporal separations, by constructing the Wilson loops, whenever possible, from thermally averaged temporal links [11]. The thermal averaging was accomplished using the Cabibbo-Marinari pseudo-heat-bath method (40 updates). Other relevant run parameters are given in Table II.

The values of the potential $V(\vec{r})$ were extracted from the Wilson loop measurements by fitting $W(\vec{r}, t)$ to the exponential form $Z(\vec{r}) \exp [-t V(\vec{r})]$ in the range $t=t_{\min }, \ldots, t_{\max }$, for each $\vec{r}$. The plateau region from $t_{\min }$ to $t_{\max }$ was chosen separately for each $\vec{r}$ in order to minimize the uncertainty in the extracted values for $V(\vec{r})$ while maintaining a good quality $Q$ of fit. Best-fit values were determined using the standard $\chi^{2}$ test, taking into account temporal correlations among the $W(\vec{r}, t)$. The covariance matrix in $\chi^{2}$ was determined using the jackknife procedure, and estimates for the uncertainties in the extracted values for $V(\vec{r})$ were computed 


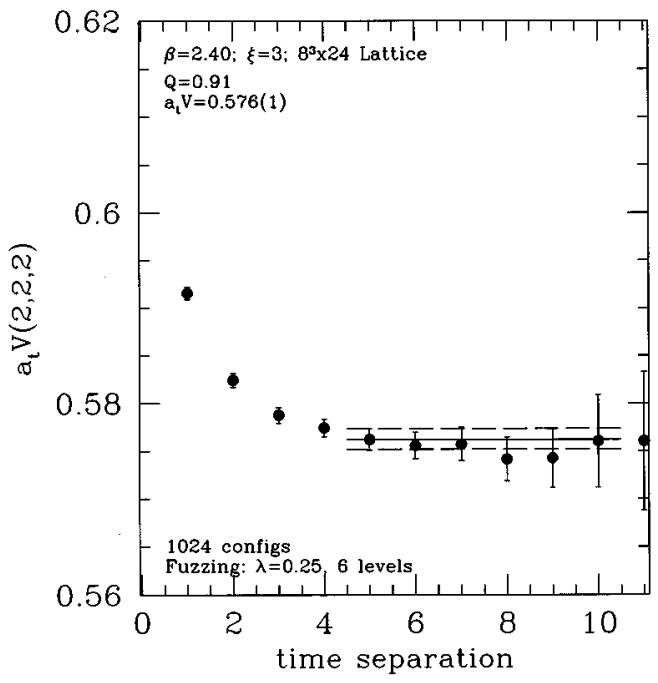

FIG. 2. Effective mass plot showing the results of a singleexponential fit to the Wilson loop for $V(\vec{r})$ with $\vec{r} / a_{s}=(2,2,2)$, $\beta=2.4$, and $\xi=3$. The $t_{\min }-t_{\max }$ region of the fit is also indicated.

using the bootstrap method. Binning of the data was done as a crude check that our measurements were statistically independent. The results of a typical fit are shown in Fig. 2, which is an effective mass plot for $V(\vec{r})$ for $\vec{r} / a_{s}=(2,2,2)$. The effective mass for $V(\vec{r})$ is a function of $t$, defined as $\ln \left[W(\vec{r}, t) / W\left(\vec{r}, t+a_{t}\right)\right]$, which tends to the true mass as $t$ becomes large.

Once a suitable plateau region in the effective mass was established for each $V(\vec{r})$, the hadronic scale $r_{0} / a_{s}$ could be determined. We found that the on-axis potential $V(\vec{r})$ for the range of $\vec{r}$ values studied here using coarse lattices fits a Coulomb plus linear form $V(\vec{r})=e_{c} / r+\sigma r+V_{0}$ very well (with qualities of fit ranging from $Q=0.25$ to $Q=0.99$ ). We, therefore, used this form to interpolate $V(\vec{r})$ and the force between static quarks. Simultaneous fits of the Wilson loops for the on-axis potential to the form $Z(r) \exp \left[-t\left(e_{c} / r+\sigma r+V_{0}\right)\right]$ were done, taking into account all correlations among the $W(r, t)$ for both different $t$ and $r$. Different regions in $t$ were used for different $r$ values; the plateau regions determined previously were used. Only the on-axis potentials were used; this prevented the covariance

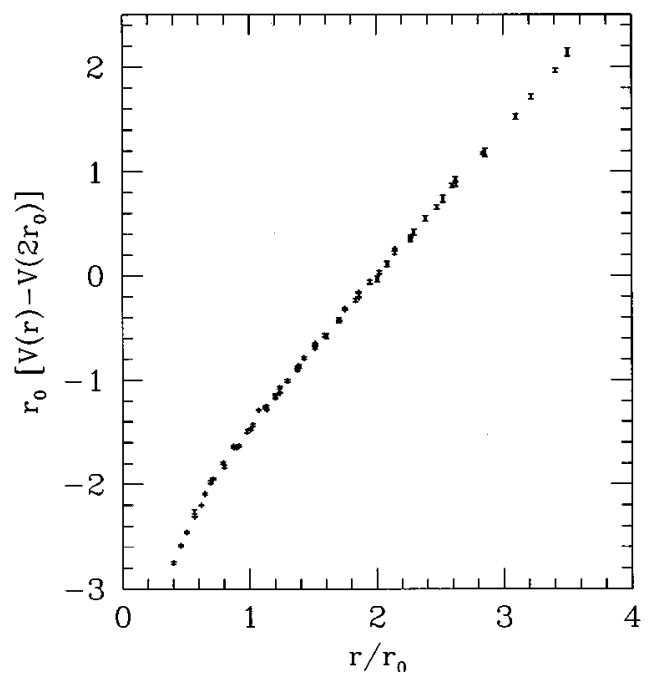

FIG. 3. The static-quark potential $V(\vec{r})$ expressed in terms of the hadronic scale $r_{0}$. This plot includes measurements from the $\beta=2.2,2.4$, and 2.6 simulations for $\xi=3$, and the $\beta=2.2$ and 2.4 simulations for $\xi=5$. Lattice spacing errors are seen to be small.

matrix in the $\chi^{2}$ to be minimized from getting too large. This covariance matrix was evaluated using the jackknife method; uncertainties in the fit parameters $e_{c}, \sigma, V_{0}$, and $Z(r)$ were obtained using the bootstrap method. Once we had an ensemble of bootstrap estimates for these fit parameters, the ratio $r_{0} / a_{s}$ and its bootstrap uncertainty were then determined using

$$
r_{0} / a_{s}=\sqrt{\left(1.65+e_{c}\right) / \sigma a_{s}^{2}}
$$

Note that to compute $r_{0} / a_{s}$, we need the ratio $a_{s} / a_{t}$ since our fits yielded estimates of $a_{t} V(\vec{r})$ only. We used the input value $\xi$ since we know that its renormalization is small. Results for $r_{0} / a_{s}$ are given in Table III.

Using the results in Table III, we can now express all energies measured in simulations in terms of $r_{0}$. For example, in Fig. 3, we show the potential, including off-axis interquark separations, expressed in terms of $r_{0}$. Lattice spacing errors are seen to be small.

TABLE III. Results for the hadronic scale $r_{0}$ in terms of the lattice spacing $a_{s}$. The Coulombic coupling $e_{c}$ and the string tension $\sigma$ obtained from a fit of the on-axis potential to a Coulomb plus linear form $V(\vec{r})=e_{c} / r+\sigma r+V_{0}$ are also given.

\begin{tabular}{llllll}
\hline \hline$\beta$ & $\xi$ & $r_{0} / a_{s}$ & \multicolumn{1}{c}{$a_{s} / r_{0}$} & \multicolumn{1}{c}{$e_{c}$} & $r_{0}^{2} \sigma$ \\
\hline 1.7 & 3 & $1.161(2)$ & $0.861(2)$ & $-0.07(1)$ & $1.58(1)$ \\
1.9 & 3 & $1.293(3)$ & $0.773(2)$ & $-0.13(2)$ & $1.52(2)$ \\
2.0 & 3 & $1.375(1)$ & $0.7271(8)$ & $-0.188(7)$ & $1.462(7)$ \\
2.2 & 3 & $1.615(2)$ & $0.6192(8)$ & $-0.288(8)$ & $1.362(8)$ \\
2.4 & 3 & $1.978(6)$ & $0.505(1)$ & $-0.321(6)$ & $1.329(6)$ \\
2.6 & 3 & $2.487(5)$ & $0.4021(9)$ & $-0.310(2)$ & $1.340(2)$ \\
1.7 & 5 & $1.224(1)$ & $0.8169(9)$ & $-0.177(9)$ & $1.473(9)$ \\
1.9 & 5 & $1.375(2)$ & $0.727(1)$ & $-0.20(1)$ & $1.45(1)$ \\
2.2 & 5 & $1.761(2)$ & $0.5680(5)$ & $-0.294(4)$ & $1.356(4)$ \\
2.4 & 5 & $2.180(6)$ & $0.459(1)$ & $-0.308(4)$ & $1.342(4)$ \\
\hline \hline
\end{tabular}


TABLE IV. Results from fits to the $\beta=1.7, \xi=3$ glueball correlators. $N_{\exp }$ is the number of exponentials used in each fit, and the fit range refers to the temporal separations $t_{\min }-t_{\max }$ used in each fit. $Q$ is the standard quality of fit. Energies are given in $a_{t}^{-1}$. Final mass estimates are highlighted in boldface.

\begin{tabular}{lccccll}
\hline \hline Channel & $N_{\text {exp }}$ & Fit range & $\chi^{2} / N_{\mathrm{DF}}$ & $Q$ & Energies & Overlaps \\
\hline$A_{1}^{++}$ & 1 & $0-5$ & 0.71 & 0.58 & $1.061(7)$ & $1.000(4)$ \\
& 1 & $1-5$ & 0.83 & 0.48 & $\mathbf{1 . 0 5}(\mathbf{2})$ & $0.99(2)$ \\
& 1 & $2-5$ & 1.20 & 0.30 & $1.04(5)$ & $0.96(9)$ \\
$E^{++}$ & 1 & $0-3$ & 0.02 & 0.98 & $1.653(8)$ & $1.000(3)$ \\
& 1 & $1-4$ & 0.12 & 0.88 & $\mathbf{1 . 6 5 ( 5 )}$ & $1.00(5)$ \\
& 2 & $1-3$ & 1.37 & 0.25 & $1.65(5)$ & $0.98(5)$ \\
& & & & $2.5(2)$ & $1.1(2)$ \\
$T_{2}^{++}$ & 1 & $0-3$ & 0.60 & 0.55 & $1.99(1)$ & $1.000(2)$ \\
& 1 & $1-3$ & 0.06 & 0.81 & $\mathbf{1 . 9 2 ( 6 )}$ & $0.94(6)$ \\
& 2 & $1-3$ & 0.65 & 0.58 & $1.90(6)$ & $0.88(8)$ \\
$T_{1}^{+-}$ & & & & $2.5(3)$ & $0.8(3)$ \\
\hline \hline
\end{tabular}

\section{RESULTS AND DISCUSSION}

\section{A. Glueball mass measurements}

To allow clear resolution of the scaling properties of the low-lying glueball masses in the improved action, two sets of simulations were performed at two different anisotropies: six lattice spacings for an aspect ratio $\xi=3$ and four spacings for $\xi=5$ were studied. The input parameters used in these simulations are given in Table I.

The results of fitting the variationally optimized correlators $C(t)$ to the functions given in Eqs. (12) and (13) are summarized in Tables IV-XIII. Effective mass plots for the two smallest- $a_{s}$ simulations are presented in Figs. 4-7 and Figs. 8-11 for $\xi=3$ and $\xi=5$, respectively. For each channel in each of the ten simulations, it was possible to find a fit region $t_{\min }-t_{\max }$ in which the correlation function was well described by its asymptotic form as indicated by the quality of fit. In other words, convincing plateaus were observed in all effective masses. The most impressive plateau, observed in the $A_{1}^{++}$channel for $\beta=2.4$ and $\xi=5$, spanned ten time slices. In most cases, the onset of the plateau occurred when the source and sink operators were separated by only one time step. The overlaps with the lowest-lying states were also found to be extremely good, better than $90 \%$ in most cases and often consistent with unity. This clearly demonstrates the effectiveness of the link-smearing and variational techniques in diminishing excited-state contamination. Fits using $t_{\min }=2$ or 0 were also done to check for agreement with the $t_{\min }=1$ results. At time separations for which the ground state could be reliably observed, the off-diagonal elements of the reduced correlation matrices $C_{A B}(t)$ were found to be consistent with zero within statistical uncertainty. This suggests that the link-smearing, variational method also gives an excellent construction of the firstexcited state in each channel.

Our best estimates for the glueball masses in terms of $a_{t}^{-1}$ are indicated in boldface in each of the Tables IV-XIII.

TABLE V. Results from fits to the $\beta=1.9, \xi=3$ glueball correlators (see Table IV).

\begin{tabular}{lccclll}
\hline \hline Channel & $N_{\text {exp }}$ & Fit range & $\chi^{2} / N_{\text {DF }}$ & $Q$ & Energies & Overlaps \\
\hline$A_{1}^{++}$ & 1 & $0-4$ & 1.05 & 0.37 & $0.878(6)$ & $1.000(5)$ \\
& 1 & $1-4$ & 1.46 & 0.23 & $\mathbf{0 . 8 7 ( 1 )}$ & $0.99(1)$ \\
& 2 & $1-3$ & 0.74 & 0.53 & $0.88(1)$ & $1.00(1)$ \\
& & & & & $1.8(1)$ & $1.0(1)$ \\
$E^{++}$ & 1 & $0-4$ & 0.11 & 0.95 & $1.493(9)$ & $1.000(3)$ \\
& 1 & $1-4$ & 0.04 & 0.96 & $\mathbf{1 . 4 7 ( 4 )}$ & $0.98(3)$ \\
& 2 & $1-3$ & 0.06 & 0.98 & $1.47(4)$ & $0.95(5)$ \\
& & & & & $1.9(1)$ & $0.91(9)$ \\
$T_{2}^{++}$ & 1 & $0-3$ & 1.74 & 0.18 & $1.681(8)$ & $1.000(2)$ \\
& 1 & $1-3$ & 0.22 & 0.64 & $\mathbf{1 . 6 0 ( 4 )}$ & $0.92(4)$ \\
& 2 & $1-3$ & 0.23 & 0.88 & $1.58(4)$ & $0.88(4)$ \\
$T_{1}^{+-}$ & & & & $2.6(2)$ & $1.3(3)$ \\
\hline \hline
\end{tabular}


TABLE VI. Results from fits to the $\beta=2.0, \xi=3$ glueball correlators (see Table IV).

\begin{tabular}{lcccccc}
\hline \hline Channel & $N_{\text {exp }}$ & Fit range & $\chi^{2} / N_{\mathrm{DF}}$ & $Q$ & Energies & Overlaps \\
\hline$A_{1}^{++}$ & 1 & $0-6$ & 0.94 & 0.45 & $0.794(4)$ & $1.000(4)$ \\
& 1 & $1-6$ & 1.15 & 0.33 & $\mathbf{0 . 7 9 7 ( 9 )}$ & $1.002(9)$ \\
& 2 & $1-3$ & 2.33 & 0.07 & $0.794(8)$ & $1.001(8)$ \\
& & & & $1.61(5)$ & $0.95(5)$ \\
$E^{++}$ & 1 & $0-4$ & 0.67 & 0.57 & $1.423(6)$ & $1.000(2)$ \\
& 1 & $1-4$ & 0.42 & 0.65 & $\mathbf{1 . 4 0 ( 2 )}$ & $0.97(2)$ \\
& 1 & $1-3$ & 1.30 & 0.27 & $1.39(2)$ & $0.96(2)$ \\
& & & & $1.81(6)$ & $0.94(5)$ \\
$T_{2}^{++}$ & 1 & $0-4$ & 2.54 & 0.05 & $1.559(5)$ & $1.000(2)$ \\
& 1 & $1-4$ & 0.67 & 0.51 & $\mathbf{1 . 4 9 ( 2 )}$ & $0.94(2)$ \\
& 2 & $1-3$ & 0.53 & 0.66 & $1.49(2)$ & $0.94(2)$ \\
& & & & $2.11(7)$ & $1.01(7)$ \\
$T_{1}^{+-}$ & 1 & $1-4$ & 0.02 & 0.98 & $\mathbf{1 . 6 8 ( 3 )}$ & $0.92(3)$ \\
\hline \hline
\end{tabular}

These estimates are summarized in Table XIV. Masses for the first-excited states are also indicated in the $N_{\exp }=2$ fits listed in the Tables IV-XIII.

\section{B. Finite volume effects}

In this work, we were concerned with the magnitude of discretization errors in the glueball mass determinations from coarse lattice simulations using an improved action. In order to evaluate these errors, we had to eliminate uncertainties from all other sources. The increased efficiency of simulations on coarse, anisotropic lattices allowed us to reduce statistical errors to the acceptable level of about $1 \%$. The only remaining source of uncertainty we had to address was the finite volume. The masses of particles confined in a small box with periodic boundary conditions can differ appreciably from their infinite-volume values; finite-volume effects can also induce a splitting in the masses of the $E$ and $T_{2}$ tensor polarizations. Finite-volume effects on the scalar glueball mass have been analyzed before [12], but the effects on the tensor and the pseudovector are less well known.

In order to ascertain the effects on our glueball masses of simulating in a finite volume, four extra simulations were performed for $\beta=2.4, \xi=3$ using lattices of spatial extent $L_{s} / a_{s}=6,5,4$, and 3 . The temporal extent was held fixed at 24 grid points. For each of these volumes, the mean-field renormalization parameter $u_{s}$ was recalculated. The $3^{3}$ lattice was the only simulation that required any change in this parameter, and in this case, the effect was small; $u_{s}$ increased by only $0.3 \%$. The results from the $L_{s} / a_{s}=8,6,5$, and 4 runs for the glueball masses in terms of $a_{t}^{-1}$ are given in Table $\mathrm{XV}$. Note that the results from the $6^{3}$ lattice differ very little from those from the $8^{3}$ lattice, suggesting that our lattice volumes are sufficiently large to ensure that finite-volume errors are negligible. For the $3^{3}$ lattice, no plateaus in the effective masses for the $A_{1}^{++}, E^{++}$, and $T_{2}^{++}$channels were observed; the mass in the $T_{1}^{+-}$channel was found to be

TABLE VII. Results from fits to the $\beta=2.2, \xi=3$ glueball correlators (see Table IV).

\begin{tabular}{|c|c|c|c|c|c|c|}
\hline Channel & $N_{\exp }$ & Fit range & $\chi^{2} / N_{\mathrm{DF}}$ & $Q$ & Energies & Overlaps \\
\hline \multirow{4}{*}{$A_{1}^{++}$} & 1 & $0-7$ & 1.12 & 0.35 & $0.659(4)$ & $0.998(6)$ \\
\hline & 1 & $1-7$ & 0.76 & 0.58 & $0.649(8)$ & $0.988(8)$ \\
\hline & 2 & $1-4$ & 1.02 & 0.41 & $0.647(8)$ & $0.984(8)$ \\
\hline & & & & & $1.25(3)$ & $0.93(2)$ \\
\hline \multirow[t]{4}{*}{$E^{++}$} & 1 & $1-4$ & 0.10 & 0.90 & $1.19(2)$ & $0.95(2)$ \\
\hline & 1 & $2-4$ & 0.10 & 0.75 & $1.17(6)$ & $0.9(1)$ \\
\hline & 2 & $1-3$ & 0.32 & 0.81 & $1.19(2)$ & $0.95(2)$ \\
\hline & & & & & $1.62(4)$ & $0.99(4)$ \\
\hline \multirow[t]{5}{*}{$T_{2}^{++}$} & 1 & $0-4$ & 2.32 & 0.07 & $1.280(6)$ & $1.001(2)$ \\
\hline & 1 & $1-4$ & 0.92 & 0.40 & $1.24(2)$ & $0.96(2)$ \\
\hline & 1 & $2-4$ & 0.02 & 0.88 & $1.16(6)$ & $0.8(1)$ \\
\hline & 2 & $1-3$ & 1.30 & 0.27 & $1.24(2)$ & $0.96(2)$ \\
\hline & & & & & $1.65(5)$ & $0.87(4)$ \\
\hline$T_{1}^{+-}$ & 1 & $1-4$ & 1.13 & 0.32 & $1.48(3)$ & $0.98(3)$ \\
\hline
\end{tabular}


TABLE VIII. Results from fits to the $\beta=2.4, \xi=3$ glueball correlators (see Table IV).

\begin{tabular}{|c|c|c|c|c|c|c|}
\hline Channel & $N_{\text {exp }}$ & Fit range & $\chi^{2} / N_{\mathrm{DF}}$ & $Q$ & Energies & Overlaps \\
\hline \multirow[t]{4}{*}{$A_{1}^{++}$} & 1 & $1-8$ & 0.19 & 0.98 & $0.548(6)$ & $0.988(6)$ \\
\hline & 1 & $2-8$ & 0.22 & 0.96 & $0.550(9)$ & $0.99(2)$ \\
\hline & 2 & $1-4$ & 1.12 & 0.35 & $0.550(6)$ & $0.991(6)$ \\
\hline & & & & & $1.03(2)$ & $0.96(1)$ \\
\hline \multirow[t]{4}{*}{$E^{++}$} & 1 & $0-5$ & 1.30 & 0.27 & $1.012(4)$ & $1.000(3)$ \\
\hline & 1 & $1-5$ & 0.31 & 0.82 & $0.995(9)$ & $0.982(9)$ \\
\hline & 2 & $1-3$ & 2.14 & 0.09 & $0.993(8)$ & $0.982(8)$ \\
\hline & & & & & $1.37(2)$ & $1.00(2)$ \\
\hline \multirow[t]{4}{*}{$T_{2}^{++}$} & 1 & $0-5$ & 3.98 & 0.00 & $1.035(4)$ & $1.001(2)$ \\
\hline & 1 & $1-5$ & 0.28 & 0.84 & $1.006(8)$ & $0.969(9)$ \\
\hline & 2 & $1-3$ & 0.53 & 0.66 & $1.006(8)$ & $0.966(8)$ \\
\hline & & & & & $1.42(2)$ & $0.99(2)$ \\
\hline$T_{1}^{+-}$ & 1 & $1-4$ & 0.75 & 0.47 & $1.24(1)$ & $0.98(1)$ \\
\hline
\end{tabular}

1.44(1). The operators used in these runs were the same as those constructed for the large-volume runs and thus, were not optimized to give large overlaps with the light torelon states (flux excitations encircling the toroidal lattice) present in small volumes. It is likely that this effect was responsible for the poor overlap of our operators with the scalar and tensor states on the $3^{3}$ lattice.

The properties, such as the mass, of a glueball confined in a small box with periodic boundary conditions differ from those in an infinite volume. The modification of the mass of a particle due to finite volume effects has been estimated in Ref. [13]:

$$
a_{t} m_{G}(z)=a_{t} m_{G}(\infty)\left[1-\lambda_{G} \exp (-\sqrt{3} z / 2) / z\right]
$$

where $z$ is the dimensionless length scale $z=m_{A_{1}^{++}} L_{s}, m_{A_{1}^{++}}$ is the infinite-volume mass of the scalar glueball, and $\lambda_{G}$ is related to the strength of an effective triple scalar glueball interaction vertex. The mass shift given in Eq. (16) is valid for sufficiently large $z$ and arises from the exchange of scalar glueballs across the periodic boundaries of the lattice. Finitevolume errors in our glueball masses measured on an $8^{3}$ lattice at $\beta=2.4, \xi=3$ (where $L_{s} \approx 2 \mathrm{fm}$, similar to the volumes used in the other nine simulations) can be estimated by fitting the form given in Eq. (16) to the masses in Table XV. Let $\omega=a_{t} m_{A_{1}^{++}}$and $\rho=\xi L_{s} / a_{s}$, then $z=\rho \omega$. The $A_{1}^{++}$fit was done first using the function $a_{t} m(z)=\omega$ $-\lambda_{A_{1}^{+}} \exp (-\sqrt{3} \rho \omega / 2) / \rho$, where $\omega$ and $\lambda_{A_{1}^{++}}$were the fitting parameters. The best-fit value for $\omega$ was then used in the fits to the results for the other irreps; to simplify matters, the uncertainty in $\omega$ was neglected in these fits. The $T_{1}^{+-}$fit also included the energy estimate extracted from the $L_{s} / a_{s}=3$ simulation. The results of these fits are summarized in Table $\mathrm{XVI}$; the estimates of the finite-volume errors are listed in the final column of this table and are given by $m_{G}(8 \omega \xi) / m_{G}(\infty)-1$ using Eq. (16). In all cases, these errors were insignificant compared to the statistical errors; this means, for example, that any differences between the large-

TABLE IX. Results from fits to the $\beta=2.6, \xi=3$ glueball correlators (see Table IV).

\begin{tabular}{lccclll}
\hline \hline Channel & $N_{\text {exp }}$ & Fit range & $\chi^{2} / N_{\text {DF }}$ & $Q$ & Energies & \multicolumn{1}{c}{ Overlaps } \\
\hline$A_{1}^{++}$ & 1 & $1-10$ & 0.88 & 0.53 & $\mathbf{0 . 4 6 4 ( 7 )}$ & $0.986(8)$ \\
& 1 & $2-10$ & 1.01 & 0.42 & $0.46(1)$ & $0.98(2)$ \\
& 2 & $1-4$ & 0.89 & 0.50 & $0.464(6)$ & $0.988(8)$ \\
& & & & & $0.84(1)$ & $0.96(1)$ \\
$E^{++}$ & 1 & $1-6$ & 0.60 & 0.67 & $\mathbf{0 . 7 8 1 ( 8 )}$ & $0.984(8)$ \\
& 1 & $2-6$ & 0.36 & 0.78 & $0.76(2)$ & $0.94(4)$ \\
& 2 & $1-4$ & 0.89 & 0.50 & $0.782(9)$ & $0.982(9)$ \\
& & & & & $1.09(2)$ & $0.95(2)$ \\
$T_{2}^{++}$ & 1 & $1-6$ & 0.31 & 0.87 & $\mathbf{0 . 7 7 7 ( 8 )}$ & $0.977(8)$ \\
& 1 & $2-6$ & 0.12 & 0.95 & $0.76(2)$ & $0.95(3)$ \\
& 2 & $1-4$ & 0.53 & 0.79 & $0.777(8)$ & $0.976(8)$ \\
$T_{1}^{+-}$ & & & & $1.12(1)$ & $0.99(1)$ \\
\hline \hline
\end{tabular}


TABLE X. Results from fits to the $\beta=1.7, \xi=5$ glueball correlators (see Table IV).

\begin{tabular}{|c|c|c|c|c|c|c|}
\hline Channel & $N_{\text {exp }}$ & Fit range & $\chi^{2} / N_{\mathrm{DF}}$ & $Q$ & Energies & Overlaps \\
\hline \multirow[t]{4}{*}{$A_{1}^{++}$} & 1 & $0-5$ & 1.36 & 0.24 & $0.585(3)$ & $0.999(4)$ \\
\hline & 1 & $1-5$ & 0.26 & 0.86 & $0.578(5)$ & $0.992(5)$ \\
\hline & 2 & $1-4$ & 0.38 & 0.89 & $0.578(5)$ & $0.992(5)$ \\
\hline & & & & & $1.19(2)$ & $0.97(2)$ \\
\hline \multirow[t]{4}{*}{$E^{++}$} & 1 & $0-5$ & 2.09 & 0.08 & $0.943(3)$ & $1.000(2)$ \\
\hline & 1 & $1-5$ & 0.12 & 0.95 & $0.924(8)$ & $0.981(7)$ \\
\hline & 2 & $1-4$ & 0.29 & 0.94 & $0.924(8)$ & $0.979(7)$ \\
\hline & & & & & $1.29(2)$ & $0.98(1)$ \\
\hline \multirow[t]{4}{*}{$T_{2}^{++}$} & 1 & $0-5$ & 1.25 & 0.29 & $1.107(3)$ & $1.001(2)$ \\
\hline & 1 & $1-5$ & 1.58 & 0.19 & $1.103(8)$ & $0.997(9)$ \\
\hline & 2 & $1-4$ & 0.87 & 0.52 & $1.104(9)$ & $0.997(9)$ \\
\hline & & & & & $1.41(2)$ & $0.94(2)$ \\
\hline \multirow[t]{4}{*}{$T_{1}^{+-}$} & 1 & $0-3$ & 2.65 & 0.07 & $1.214(4)$ & $1.000(2)$ \\
\hline & 1 & $1-3$ & 0.21 & 0.65 & 1.19(1) & $0.97(1)$ \\
\hline & 2 & $1-3$ & 0.39 & 0.76 & $1.18(1)$ & $0.97(1)$ \\
\hline & & & & & $1.55(3)$ & $0.92(2)$ \\
\hline
\end{tabular}

volume masses in the $T_{2}$ and $E$ channels must be due purely to discretization errors. It is interesting to note that our estimate of $\lambda_{A_{1}^{+}}$agrees well with the value $190 \pm 70$ found in Ref. [12].

\section{Continuum limit extrapolations}

The glueball mass estimates in terms of $a_{t}^{-1}$ were combined with the determinations of the hadronic scale $r_{0} / a_{s}$. The results are shown in Figs. 12-14. In these figures, the dimensionless product of $r_{0}$ and the glueball mass estimates are shown as functions of $\left(a_{s} / r_{0}\right)^{2}$. Solid symbols indicate results from the $\xi=3$ simulations, while open symbols are used for the results from the $\xi=5$ runs. In Fig. 12, the lowest-lying masses in each of the channels $A_{1}^{++}, E^{++}$, $T_{2}^{++}$, and $T_{1}^{+-}$are compared with results from small- $a_{s}$ Wilson action simulations. The lowest-lying and first-excited masses in the $E^{++}$and $T_{2}^{++}$channels are shown in Fig. 13, and the ground state and first-excited state in the $A_{1}^{++}$channel are depicted in Fig. 14. To extract physical predictions (for the pure-gauge theory), the curves in these plots must be extrapolated to the continuum limit $a_{s} / r_{0} \rightarrow 0$. Discretization errors are given by the deviations of the finite- $a_{s}$ results from these limiting values.

The lowest-lying states in the $E^{++}$and $T_{2}^{++}$channels correspond to the five polarizations of the tensor $2^{++}$glueball in the continuum. Differences between the $E^{++}$and $T_{2}^{++}$masses are a measure of violations of rotational sym-

TABLE XI. Results from fits to the $\beta=1.9, \xi=5$ glueball correlators (see Table IV).

\begin{tabular}{|c|c|c|c|c|c|c|}
\hline Channel & $N_{\text {exp }}$ & Fit range & $\chi^{2} / N_{\mathrm{DF}}$ & $Q$ & Energies & Overlaps \\
\hline \multirow[t]{4}{*}{$A_{1}^{++}$} & 1 & $1-9$ & 1.26 & 0.26 & $0.475(4)$ & $0.992(5)$ \\
\hline & 1 & $2-9$ & 1.11 & 0.35 & $0.468(6)$ & $0.98(1)$ \\
\hline & 2 & $2-4$ & 1.03 & 0.38 & $0.468(6)$ & $0.98(1)$ \\
\hline & & & & & $0.92(3)$ & $0.85(5)$ \\
\hline \multirow[t]{4}{*}{$E^{++}$} & 1 & $1-6$ & 0.42 & 0.80 & $0.844(6)$ & $0.992(6)$ \\
\hline & 1 & $2-6$ & 0.20 & 0.90 & $0.83(1)$ & $0.97(2)$ \\
\hline & 2 & $1-4$ & 0.69 & 0.66 & $0.844(6)$ & $0.992(6)$ \\
\hline & & & & & $1.09(1)$ & $0.95(1)$ \\
\hline \multirow[t]{4}{*}{$T_{2}^{++}$} & 1 & $1-5$ & 0.91 & 0.43 & $0.918(7)$ & $0.982(6)$ \\
\hline & 1 & $2-5$ & 1.11 & 0.33 & $0.91(2)$ & $0.96(3)$ \\
\hline & 2 & $1-4$ & 1.44 & 0.19 & $0.918(6)$ & $0.981(6)$ \\
\hline & & & & & $1.23(1)$ & $0.96(1)$ \\
\hline \multirow[t]{4}{*}{$T_{1}^{+-}$} & 1 & $1-5$ & 0.30 & 0.83 & $1.053(8)$ & $0.979(7)$ \\
\hline & 1 & $2-5$ & 0.18 & 0.84 & $1.04(2)$ & $0.95(4)$ \\
\hline & 2 & $1-4$ & 0.48 & 0.82 & $1.052(9)$ & $0.97(1)$ \\
\hline & & & & & $1.30(1)$ & $0.92(1)$ \\
\hline
\end{tabular}


TABLE XII. Results from fits to the $\beta=2.2, \xi=5$ glueball correlators (see Table IV).

\begin{tabular}{|c|c|c|c|c|c|c|}
\hline Channel & $N_{\text {exp }}$ & Fit range & $\chi^{2} / N_{\mathrm{DF}}$ & $Q$ & Energies & Overlaps \\
\hline \multirow[t]{4}{*}{$A_{1}^{++}$} & 1 & $1-14$ & 0.60 & 0.84 & $0.362(3)$ & $0.998(5)$ \\
\hline & 1 & $2-14$ & 0.50 & 0.90 & $0.366(4)$ & $1.004(7)$ \\
\hline & 2 & $1-4$ & 0.86 & 0.52 & $0.362(3)$ & $0.998(5)$ \\
\hline & & & & & $0.697(6)$ & $0.970(7)$ \\
\hline \multirow[t]{4}{*}{$E^{++}$} & 1 & $1-7$ & 1.33 & 0.25 & $0.667(4)$ & $0.982(4)$ \\
\hline & 1 & $2-7$ & 1.65 & 0.16 & $0.666(7)$ & $0.98(1)$ \\
\hline & 2 & $1-4$ & 0.57 & 0.75 & $0.667(4)$ & $0.980(5)$ \\
\hline & & & & & $0.878(7)$ & $0.968(7)$ \\
\hline \multirow[t]{4}{*}{$T_{2}^{++}$} & 1 & $1-8$ & 1.05 & 0.39 & $0.686(4)$ & $0.983(3)$ \\
\hline & 1 & $2-8$ & 1.20 & 0.31 & $0.683(6)$ & $0.98(1)$ \\
\hline & 2 & $1-4$ & 0.77 & 0.59 & $0.686(3)$ & $0.982(3)$ \\
\hline & & & & & $0.938(5)$ & $0.970(5)$ \\
\hline \multirow[t]{4}{*}{$T_{1}^{+-}$} & 1 & $1-6$ & 0.48 & 0.75 & $0.819(4)$ & $0.974(4)$ \\
\hline & 1 & $2-6$ & 0.57 & 0.63 & $0.82(1)$ & $0.98(2)$ \\
\hline & 2 & $1-4$ & 0.60 & 0.73 & $0.820(4)$ & $0.974(4)$ \\
\hline & & & & & $1.025(8)$ & $0.956(7)$ \\
\hline
\end{tabular}

metry due to finite spacing artifacts. In Fig. 12, such violations are seen to be small for our less coarse lattices and become appreciable as the spacing gets very large. Discretization errors in the $T_{2}^{++}$exceed those of the $E^{++}$; on our coarsest lattices, finite spacing errors are only a few percent for the $E^{++}$channel, but about $15 \%$ in the $T_{2}^{++}$channel. In the $E^{++}$channel, the $\xi=3$ results differ very little from those using the higher aspect ratio $\xi=5$, suggesting that the $O\left(a_{t}^{2}\right)$ errors are negligible. However, small differences between the results from the two anisotropies are visible in the $T_{2}^{++}$channel. One expects that $O\left(a_{t}^{2}\right)$ errors will decrease as $\xi$ is increased. Since the $T_{2}^{++}$discretization errors are slightly larger for the $\xi=5$ runs, $O\left(a_{t}^{2}\right)$ errors can account for this difference only if such errors offset the $O\left(a_{s}^{4}\right)$ errors.
The leading discretization errors in the tensor glueball masses are expected to be $O\left(a_{t}^{2}, a_{s}^{4}, \alpha_{s} a_{s}^{2}\right)$. However, we have already argued that the results in Fig. 12 imply that the $O\left(a_{t}^{2}\right)$ errors are negligible. Since the action included meanfield correction factors, we also expected that $O\left(a_{s}^{4}\right)$ errors would dominate over $O\left(\alpha_{s} a_{s}^{2}\right)$ errors and in our continuum limit extrapolations, we assumed that this was true unless the fit provided compelling evidence to the contrary. Although we expected the leading discretization errors to be $O\left(a_{s}^{4}\right)$, the following three functions were used in our continuum limit extrapolations:

$$
\begin{gathered}
\varphi_{0}\left(a_{s}\right)=r_{0} m_{G}, \\
\varphi_{2}\left(a_{s}\right)=r_{0} m_{G}+c_{2}\left(a_{s} / r_{0}\right)^{2},
\end{gathered}
$$

\begin{tabular}{|c|c|c|c|c|c|c|}
\hline Channel & $N_{\exp }$ & Fit range & $\chi^{2} / N_{\mathrm{DF}}$ & $Q$ & Energies & Overlaps \\
\hline \multirow[t]{4}{*}{$A_{1}^{++}$} & 1 & $1-13$ & 1.35 & 0.19 & $0.303(3)$ & $0.995(7)$ \\
\hline & 1 & $2-13$ & 1.15 & 0.32 & $0.307(4)$ & $1.000(8)$ \\
\hline & 2 & $1-5$ & 0.86 & 0.56 & $0.304(3)$ & $0.994(7)$ \\
\hline & & & & & $0.569(4)$ & $0.972(5)$ \\
\hline \multirow[t]{4}{*}{$E^{++}$} & 1 & $1-9$ & 1.33 & 0.23 & $0.538(3)$ & $0.992(3)$ \\
\hline & 1 & $2-9$ & 1.46 & 0.19 & $0.536(5)$ & $0.986(9)$ \\
\hline & 2 & $2-5$ & 2.71 & 0.01 & $0.538(5)$ & $0.99(1)$ \\
\hline & & & & & $0.723(9)$ & $0.94(1)$ \\
\hline \multirow[t]{4}{*}{$T_{2}^{++}$} & 1 & $1-9$ & 1.08 & 0.38 & $0.542(2)$ & $0.988(3)$ \\
\hline & 1 & $2-7$ & 0.34 & 0.85 & $0.540(4)$ & $0.982(7)$ \\
\hline & 2 & $2-5$ & 1.28 & 0.26 & $0.538(4)$ & $0.978(8)$ \\
\hline & & & & & $0.730(8)$ & $0.94(1)$ \\
\hline \multirow[t]{3}{*}{$T_{1}^{+-}$} & 1 & $2-6$ & 1.03 & 0.38 & $0.652(5)$ & $0.95(1)$ \\
\hline & 2 & $2-6$ & 1.77 & 0.07 & $0.648(6)$ & $0.95(1)$ \\
\hline & & & & & $0.794(9)$ & $0.88(2)$ \\
\hline
\end{tabular}

TABLE XIII. Results from fits to the $\beta=2.4, \xi=5$ glueball correlators (see Table IV). 


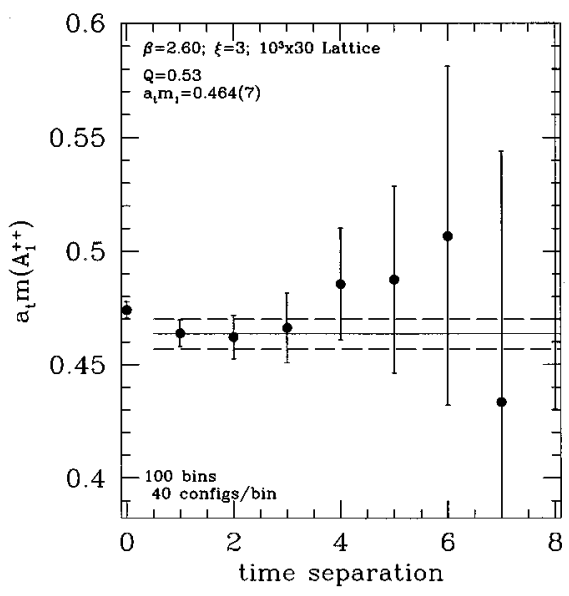

FIG. 4. Effective mass plot showing the results of a singleexponential fit to the glueball correlation function for the $A_{1}^{++}$ channel for $\beta=2.6$ and $\xi=3$. The $t_{\min }-t_{\max }$ region of the fit is also indicated.

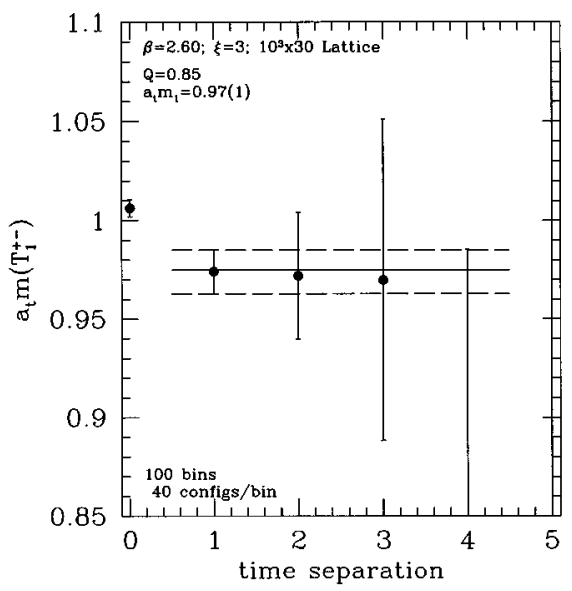

FIG. 5. Effective mass plot showing the results of a singleexponential fit to the glueball correlation function for the $T_{1}^{+-}$ channel for $\beta=2.6$ and $\xi=3$.

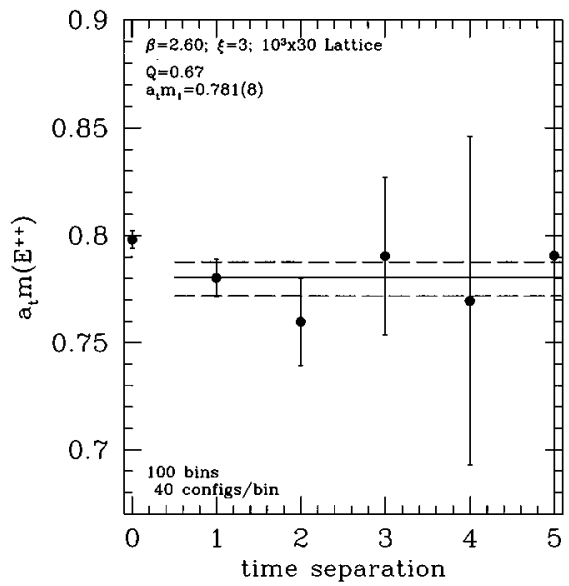

FIG. 6. Effective mass plot showing the results of a singleexponential fit to the glueball correlation function for the $E^{++}$ channel for $\beta=2.6$ and $\xi=3$.

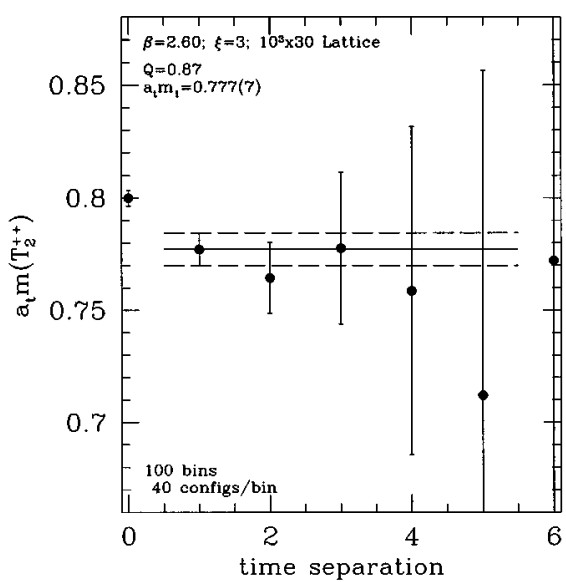

FIG. 7. Effective mass plot showing the results of a singleexponential fit to the glueball correlation function for the $T_{2}^{++}$ channel for $\beta=2.6$ and $\xi=3$.

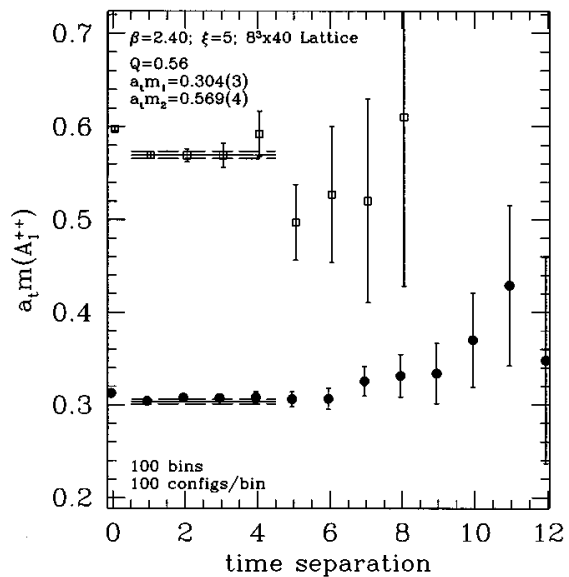

FIG. 8. Effective mass plot showing the results of a twoexponential fit to the $2 \times 2$ matrix of glueball correlation functions for the $A_{1}^{++}$channel for $\beta=2.4$ and $\xi=5$. The $t_{\min }-t_{\max }$ region of the fit is also indicated.

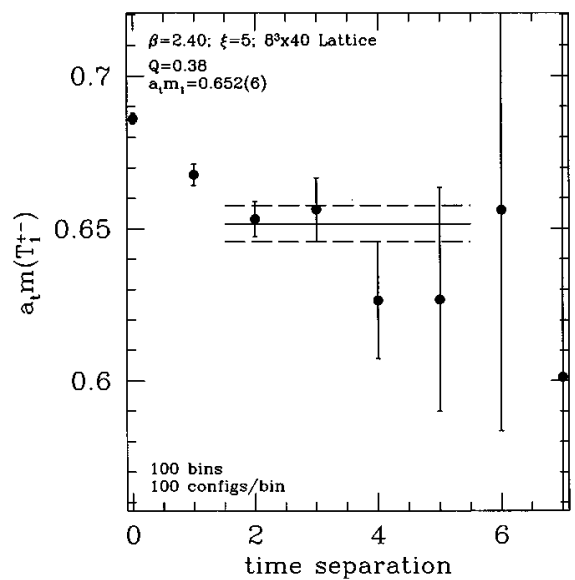

FIG. 9. Effective mass plot showing the results of a singleexponential fit to the glueball correlation function for the $T_{1}^{+-}$ channel for $\beta=2.4$ and $\xi=5$. 


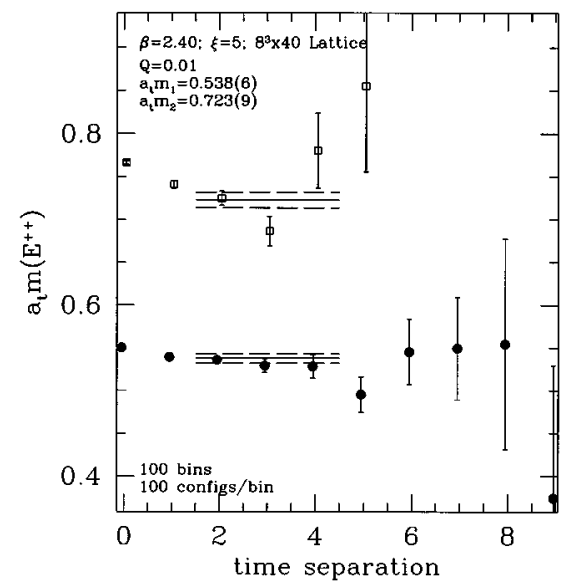

FIG. 10. Effective mass plot showing the results of a twoexponential fit to the $2 \times 2$ matrix of glueball correlation functions for the $E^{++}$channel for $\beta=2.4$ and $\xi=5$. The $t_{\min }-t_{\max }$ region of the fit is also indicated.

$$
\varphi_{4}\left(a_{s}\right)=r_{0} m_{G}+c_{4}\left(a_{s} / r_{0}\right)^{4}
$$

where $c_{2}, c_{4}$, and $r_{0} m_{G}$ are best-fit parameters. The results of these fits are given in Table XVII for the $\xi=3$ data and Table XVIII for the $\xi=5$ simulations. Comparing the values of $\chi^{2} / N_{\mathrm{DF}}$, one sees that the fitting function $\varphi_{4}$ was preferred for both the $\xi=3$ and $\xi=5$ results, although only marginally so for $\xi=3$. Given this fact and our expectation that $\varphi_{4}$ should best describe the leading discretization effects, we took $r_{0} m_{G}$ from the $\varphi_{4}$ fits as our continuum limit estimates (indicated in boldface in Tables XVII and XVIII). These four estimates are in very good agreement not only with one another, but also with the Wilson action estimates. These fits using $\varphi_{4}$ are shown in Fig. 12. For our final estimate of the tensor glueball mass, we performed a simultaneous fit with the four data sets (two irreps and two anisotropies) using four separate $\varphi_{4}$ functions but constraining the intercept parameter $r_{0} m_{G}$ to be the same for all four fitting functions. This yielded $r_{0} m\left(2^{++}\right)=5.85 \pm 0.02$ with $\chi^{2} / N_{\mathrm{DF}}=1.01$, in agreement with the Wilson action estimate $r_{0} m\left(2^{++}\right)=6.0 \pm 0.1$, obtained by fitting all of the Wilson action measurements shown in Fig. 12 to $\varphi_{0}$.

We also examined the discretization errors in the masses of the first-excited glueball states in the $E^{++}$and $T_{2}^{++}$chan-

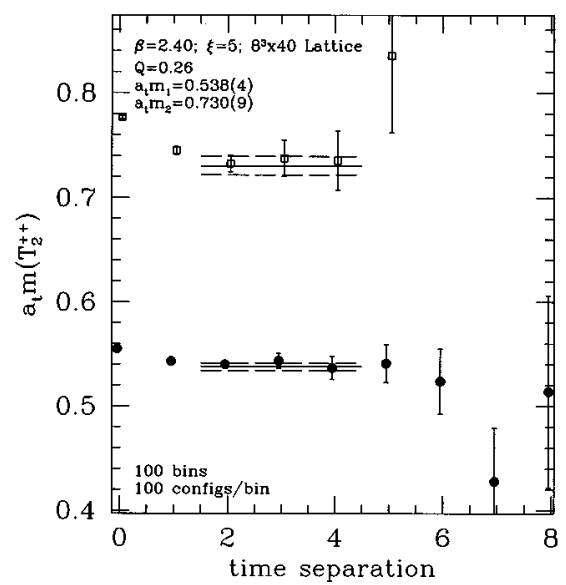

FIG. 11. Effective mass plot showing the results of a twoexponential fit to the $2 \times 2$ matrix of glueball correlation functions for the $T_{2}^{++}$channel for $\beta=2.4$ and $\xi=5$. The $t_{\min }-t_{\max }$ region of the fit is also indicated.

nels. These are shown in Fig. 13. There are several reasons for interpreting these data as different polarizations of a spintwo excited state: the two irreps extrapolate to the same continuum limit value; if the $T_{2}^{++}$state were spin three, then there would be a degeneracy with the $T_{1}^{++}$and $A_{2}^{++}$channels and this was not observed (these results will be presented elsewhere); if they were polarizations of a spin-four state, then again, a similar level must also be found in the $T_{1}^{++}$channel. The degeneracy between the two irreps and the weak finite-volume dependence of their energies also makes an interpretation of this state as a torelon pair or a two-scalar-glueball scattering state unlikely, although the mass of this level lies close to twice the mass of the scalar glueball. Continuum limit extrapolations were performed using the three functions of Eqs. (17)-(19); measurements from the two largest $a_{s}$ spacings for $\xi=3$ and the single largest spacing for $\xi=5$ were excluded from these fits. The results are given in Tables XVII and XVIII. Again, we expected $\varphi_{4}$ to provide the most reliable extrapolations to the $a_{s} \rightarrow 0$ limit; this was confirmed by the fact that $\varphi_{4}$ yielded $E^{++}$and $T_{2}^{++}$continuum limits in best agreement with each other. Differences found between the $\xi=3$ and $\xi=5$ extrapolations were not statistically significant. The fits using $\varphi_{4}$ are shown in Fig. 13. Our final determination of the mass

TABLE XIV. Summary of final mass estimates from all $\xi=3$ and $\xi=5$ simulations.

\begin{tabular}{llcccc}
\hline \hline$\beta$ & $\xi$ & $a_{t} m\left(A_{1}^{++}\right)$ & $a_{t} m\left(E^{++}\right)$ & $a_{t} m\left(T_{2}^{++}\right)$ & $a_{t} m\left(T_{1}^{+-}\right)$ \\
\hline 1.7 & 3 & $1.05(2)$ & $1.65(5)$ & $1.92(6)$ & $2.09(9)$ \\
1.9 & 3 & $0.87(1)$ & $1.47(4)$ & $1.60(4)$ & $1.80(6)$ \\
2.0 & 3 & $0.797(9)$ & $1.40(2)$ & $1.49(2)$ & $1.68(3)$ \\
2.2 & 3 & $0.649(8)$ & $1.19(2)$ & $1.24(2)$ & $1.48(3)$ \\
2.4 & 3 & $0.548(6)$ & $0.995(9)$ & $1.006(8)$ & $1.24(1)$ \\
2.6 & 3 & $0.464(7)$ & $0.781(8)$ & $0.777(8)$ & $0.97(1)$ \\
1.7 & 5 & $0.578(5)$ & $0.924(8)$ & $1.103(8)$ & $1.19(1)$ \\
1.9 & 5 & $0.475(4)$ & $0.844(6)$ & $0.918(7)$ & $1.053(8)$ \\
2.2 & 5 & $0.362(3)$ & $0.667(4)$ & $0.686(4)$ & $0.819(4)$ \\
2.4 & 5 & $0.303(3)$ & $0.538(3)$ & $0.542(2)$ & $0.652(5)$ \\
\hline \hline
\end{tabular}


TABLE XV. Glueball mass estimates in terms of $a_{t}^{-1}$ for $\beta=2.4, \xi=3$, and various lattice volumes.

\begin{tabular}{lllll}
\hline \hline Channel & $L_{s} / a_{s}=4$ & $L_{s} / a_{s}=5$ & $L_{s} / a_{s}=6$ & $L_{s} / a_{s}=8$ \\
\hline$A_{1}^{++}$ & $0.483(6)$ & $0.551(6)$ & $0.545(8)$ & $0.548(6)$ \\
$A_{1}^{*++}$ & $0.863(9)$ & $0.97(1)$ & $1.00(3)$ & $1.027(17)$ \\
$E^{++}$ & $0.848(9)$ & $0.980(10)$ & $1.004(19)$ & $0.995(9)$ \\
$E^{*++}$ & $1.11(2)$ & $1.27(2)$ & $1.41(4)$ & $1.37(2)$ \\
$T 2^{++}$ & $1.032(9)$ & $1.005(9)$ & $1.002(14)$ & $1.006(8)$ \\
$T 2^{*++}$ & $1.37(2)$ & $1.42(2)$ & $1.40(3)$ & $1.42(2)$ \\
$T 1^{+-}$ & $1.245(13)$ & $1.235(11)$ & $1.209(25)$ & $1.237(14)$ \\
\hline \hline
\end{tabular}

of the excited tensor glueball, obtained from a constrained set of four $\varphi_{4}$, similar to the ground-state extraction, was $r_{0} m\left(2^{*+}\right)=8.11 \pm 0.04$, where $\chi^{2} / N_{\mathrm{DF}}=2.3$. This mass has not been reliably determined in any previous simulation.

Finite-spacing errors in the mass of the $T_{1}^{+-}$pseudovector glueball were also studied. These were found to be small and are shown in Fig. 12. The results from the different anisotropies are in good agreement. Extrapolations to the $a_{s} \rightarrow 0$ limit were done using the three functions $\varphi_{0}, \varphi_{2}$, and $\varphi_{4}$; the results of these fits are summarized in Tables XVII and XVIII. The continuum limits obtained from fits to the $\xi=3$ and $\xi=5$ data agreed only for the constant fit form $\varphi_{0}$. The fits to $\varphi_{2}$ and $\varphi_{4}$ yielded slope parameters $\left(c_{2}\right.$ and $\left.c_{4}\right)$ with large relative errors and opposite signs for the different anisotropies. Hence, the function $\varphi_{0}$ was used to extrapolate to the continuum limit. Due to the very good agreement between the $\xi=3$ and $\xi=5$ results, all ten data points were used in our extrapolation fit. Our estimate from this fit (shown in Fig. 12) was $r_{0} m\left(1^{+-}\right)=7.21 \pm 0.02$, where $\chi^{2} / N_{\mathrm{DF}}=1.55$, in agreement with the extrapolation $r_{0} m\left(1^{+-}\right)=7.5 \pm 0.4$, using $\varphi_{0}$ of the Wilson action results shown in Fig. 12.

In contrast to the tensor and pseudovector, the scalar glueball mass showed significant finite-spacing errors (see Fig. 12), even for our less coarse lattices. As $a_{s}$ was increased, the scalar mass first decreased, reached a minimum near $a_{s} / r_{0} \sim 0.6$, then gradually increased. Near the minimum, the mass was about $25 \%$ lower than estimates of the continuum limit from small- $a_{s}$ Wilson action computations; a $20 \%$ discretization error was observed in the result from our smallest $a_{s}$ simulation. Although the magnitudes of these errors were significant, they were smaller than those obtained using the Wilson action by a factor of 2 . In order to extrapolate to the

TABLE XVI. The effects of simulating in a finite box: results from fits of Eq. (16) to the energy estimates given in Table XV from lattices of spatial extent $L_{s} / a_{s}=4,5,6,8$. The $T_{1}^{+-}$fit also includes an energy estimate for $L_{s} / a_{s}=3$. The final column estimates the expected finite-volume errors in glueball masses from the $L_{s} / a_{s}=8$ simulation at $\beta=2.4$ and $\xi=3$. These errors are estimated by $m_{G}(8 \omega \xi) / m_{G}(\infty)-1$ using Eq. (16).

\begin{tabular}{lcrcc}
\hline \hline Channel & $a_{t} m_{G}(\infty)$ & \multicolumn{1}{c}{$\lambda_{G}$} & $\chi^{2} / N_{\mathrm{DF}}$ & $\%$ correction \\
\hline$A_{1}^{++}$ & $0.554(4)$ & $260(37)$ & 2.3 & -0.020 \\
$E^{++}$ & $1.002(7)$ & $319(23)$ & 0.62 & -0.024 \\
$T_{2}^{++}$ & $1.003(6)$ & $-59(24)$ & 0.15 & +0.004 \\
$T_{1}^{+-}$ & $1.223(7)$ & $-66(4)$ & 1.05 & +0.005 \\
\hline \hline
\end{tabular}

continuum limit, an appropriate fitting function was needed. The leading discretization errors were expected to be $O\left(a_{t}^{2}, a_{s}^{4}, \alpha_{s} a_{s}^{2}\right)$. However, there were no distinguishable differences between the $\xi=3$ and $\xi=5$ results, suggesting that the $O\left(a_{t}^{2}\right)$ errors were negligible, leaving us to consider $O\left(a_{s}^{4}, \alpha_{s} a_{s}^{2}\right)$ effects. By inspection, one sees that the fitting form $\varphi_{4}$, which neglects one-loop $O\left(\alpha_{s} a_{s}^{2}\right)$ effects, cannot describe the data, in contrast to the data for the tensor and pseudovector glueballs. As $a_{s} \rightarrow 0$, we expect the coupling $\alpha_{s}\left(a_{s}\right)$ to vanish as $-1 / \ln \left(a_{s} \Lambda\right)$, where $\Lambda$ is an appropriate scale parameter. Hence, we were led to consider the following four-parameter fitting function:

$$
\varphi_{1 L}\left(a_{s}\right)=r_{0} m_{G}+c_{2} \frac{\left(a_{s} / r_{0}\right)^{2}}{c_{L}-\ln \left[\left(a_{s} / r_{0}\right)^{2}\right]}+c_{4}\left(a_{s} / r_{0}\right)^{4}
$$

However, it was not known how reliably the leading perturbative behavior of $\alpha_{s}\left(a_{s}\right)$ would describe the true cutoff dependence of the coupling over the large range of spacings considered. Taking this into account and inspecting the be-

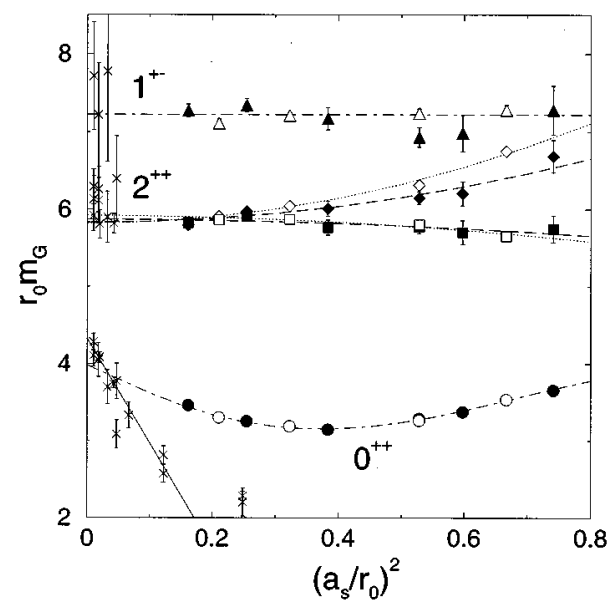

FIG. 12. Glueball mass estimates in terms of $r_{0}$ against the lattice spacing $\left(a_{s} / r_{0}\right)^{2}$. Results from the $\xi=5$ simulations for the lattice irreps $A_{1}^{++}, E^{++}, T_{2}^{++}$, and $T_{1}^{+-}$are labeled $\bigcirc, \square, \diamond$, and $\triangle$, respectively. The corresponding solid symbols indicate the results from the $\xi=3$ simulations. Data from Wilson action simulations taken from Refs. [19-22] are shown using crosses. The dashed, dotted, and dash-dotted curves indicate extrapolations to the continuum limit obtained by fitting to the $\xi=3$ data, the $\xi=5$ data, and all data, respectively. The solid line indicates the extrapolation of the Wilson action data to the continuum limit. 


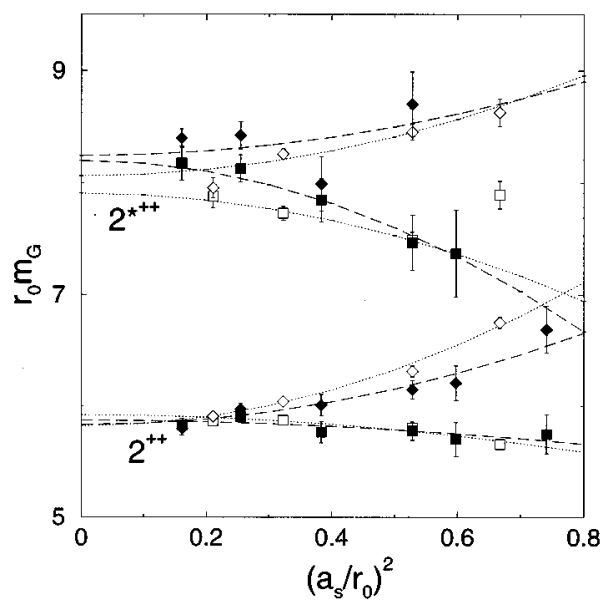

FIG. 13. Estimates of the masses of the tensor glueball and its first-excited state in terms of $r_{0}$ against the lattice spacing $\left(a_{s} / r_{0}\right)^{2}$. Results from the $\xi=5$ simulations for the $E^{++}$and $T_{2}^{++}$irreps are labeled by $\square$ and $\diamond$, respectively. The corresponding solid symbols show the results from the $\xi=3$ simulations. The dashed and dotted curves indicate extrapolations to the continuum limit obtained by fitting to the $\xi=3$ and the $\xi=5$ data, respectively (see Tables XVII and XVIII).

havior of the actual data, we decided to also consider the following simpler quadratic form:

$$
\varphi_{2,4}\left(a_{s}\right)=r_{0} m_{G}+c_{2}\left(a_{s} / r_{0}\right)^{2}+c_{4}\left(a_{s} / r_{0}\right)^{4} .
$$

Both of these functions were fit to the mass measurements from all ten simulations; the results of these fits are summarized in Table XIX. The function $\varphi_{1 L}$ yielded a slightly better fit and a continuum limit for the scalar glueball mass of 3.98 \pm 0.15 . This fit is shown in Fig. 12. An extrapolation of existing Wilson action data using $\varphi_{2}$ yielded 4.33 \pm 0.05 . Given the quality of the scalar glueball mass estimates using

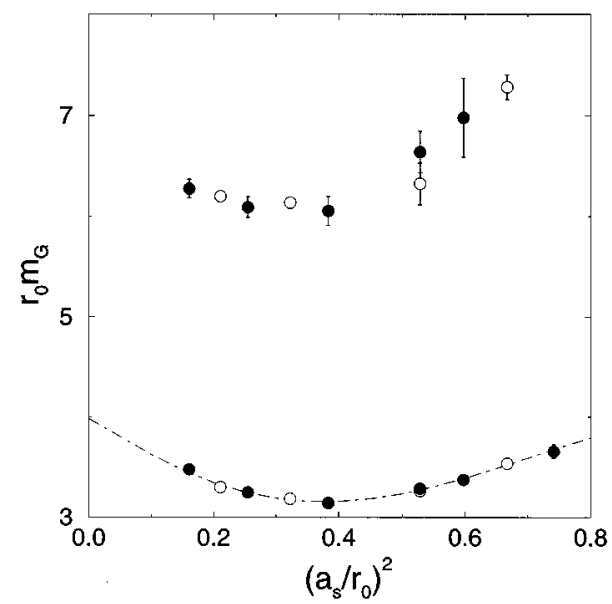

FIG. 14. Masses of the scalar glueball and its first-excited state in terms of $r_{0}$ against the lattice spacing $\left(a_{s} / r_{0}\right)^{2}$. Open and solid symbols indicate results from the $\xi=5$ and $\xi=3$ simulations, respectively. The extrapolation to the continuum limit is indicated by the dash-dotted curve.

the Wilson action, this slight discrepancy raises doubts concerning the reliability of the extrapolation using $\varphi_{1 L}$; mass estimates using the improved action for a few values of $a_{s}$ smaller than those considered here would be needed to resolve this discrepancy.

One explanation for the $20 \%$ discretization errors in the scalar glueball mass is that the scalar glueball is extremely small. However, there is evidence $[14,15]$ that the presence of a critical end point of a line of phase transitions (not corresponding to any physical transition found in QCD) in the fundamental-adjoint coupling plane is responsible for lowering the scalar glueball mass near the crossover region in the Wilson action. It is possible that the scalar glueball mass in the improved action used here may be similarly influenced. If so, the fact that this effect appears to be less

TABLE XVII. Extrapolations of the glueball mass estimates to the continuum limit for the $\xi=3$ runs. The three scaling forms $\varphi_{0}, \varphi_{2}$, and $\varphi_{4}$ which are fit to the data are given in Eqs. (17)-(19). The values indicated in bold are taken as our final continuum mass estimates.

\begin{tabular}{|c|c|c|c|c|c|}
\hline Channel & Fit function & $r_{0} m_{G}$ & $c_{2}$ & $c_{4}$ & $\chi^{2} / N_{\mathrm{DF}}$ \\
\hline \multirow[t]{3}{*}{$E^{++}$} & $\varphi_{0}$ & $5.83(3)$ & & & 0.71 \\
\hline & $\varphi_{2}$ & $5.91(7)$ & $-0.26(20)$ & & 0.49 \\
\hline & $\varphi_{4}$ & $5.87(5)$ & & $-0.33(25)$ & 0.46 \\
\hline \multirow[t]{3}{*}{$T_{2}^{++}$} & $\varphi_{0}$ & $5.98(3)$ & & & 5.35 \\
\hline & $\varphi_{2}$ & $5.66(7)$ & $1.02(21)$ & & 0.90 \\
\hline & $\varphi_{4}$ & $5.83(5)$ & & $1.29(27)$ & 0.87 \\
\hline \multirow[t]{3}{*}{$T_{1}^{+-}$} & $\varphi_{0}$ & $7.22(5)$ & & & 1.81 \\
\hline & $\varphi_{2}$ & $7.44(11)$ & $-0.71(31)$ & & 0.96 \\
\hline & $\varphi_{4}$ & $7.32(7)$ & & $-0.87(40)$ & 1.07 \\
\hline \multirow[t]{3}{*}{$E^{*++}$} & $\varphi_{0}$ & $7.99(8)$ & & & 2.44 \\
\hline & $\varphi_{2}$ & $8.52(20)$ & $-1.8(6)$ & & 0.31 \\
\hline & $\varphi_{4}$ & $8.25(12)$ & & $-2.6(9)$ & 0.12 \\
\hline \multirow[t]{3}{*}{$T_{2}^{*++}$} & $\varphi_{0}$ & $8.37(6)$ & & & 1.33 \\
\hline & $\varphi_{2}$ & $8.32(16)$ & $0.2(7)$ & & 1.94 \\
\hline & $\varphi_{4}$ & $8.34(8)$ & & $0.5(11)$ & 1.89 \\
\hline
\end{tabular}


TABLE XVIII. Extrapolations of the glueball mass estimates to the continuum limit for the $\xi=5$ runs. The three scaling forms $\varphi_{0}, \varphi_{2}$, and $\varphi_{4}$ which are fit to the data are given in Eqs. (17)-(19). The values indicated in bold are taken as our final continuum mass estimates.

\begin{tabular}{lccccc}
\hline \hline Channel & Fit function & $r_{0} m_{G}$ & $c_{2}$ & $c_{4}$ & $\chi^{2} / N_{\mathrm{DF}}$ \\
\hline$E^{++}$ & $\varphi_{0}$ & $5.82(2)$ & & & 5.03 \\
& $\varphi_{2}$ & $5.98(5)$ & $-0.41(12)$ & & 1.55 \\
& $\varphi_{4}$ & $\mathbf{5 . 9 1 ( 3 )}$ & & $-0.50(14)$ & 0.81 \\
$T_{2}^{++}$ & $\varphi_{0}$ & $6.12(2)$ & & & 82 \\
& $\varphi_{2}$ & $5.53(4)$ & $1.69(11)$ & & 5.16 \\
& $\varphi_{4}$ & $\mathbf{5 . 8 2 ( 3 )}$ & & $2.02(13)$ & 1.32 \\
$T_{1}^{+-}$ & $\varphi_{0}$ & $\mathbf{7 . 2 1}(2)$ & & & 1.61 \\
& $\varphi_{2}$ & $7.09(7)$ & $0.31(16)$ & & 0.57 \\
$\varphi_{4}^{*++}$ & $7.15(4)$ & & $0.32(18)$ & 0.80 \\
& $\varphi_{0}$ & $7.67(4)$ & & & 5.88 \\
& $\varphi_{2}$ & $8.12(14)$ & $-1.2(4)$ & & 0.02 \\
$T_{2}^{*++}$ & $\varphi_{4}$ & $\mathbf{7 . 9 1 ( 8 )}$ & & $-1.5(4)$ & 0.27 \\
& $\varphi_{0}$ & $8.29(3)$ & & & 9.17 \\
& $\varphi_{2}$ & $7.80(10)$ & $1.3(3)$ & & 1.37 \\
\hline \hline
\end{tabular}

pronounced for this action suggests the possible existence of other perturbatively improved actions in which the scalar glueball mass is even less affected by scaling violations. We are currently searching for such actions.

Discretization errors in the mass of the first-excited state in the $A_{1}^{++}$channel were also found to be significant, as shown in Fig. 14. The mass of this state is nearly twice that of the lowest-lying scalar glueball, suggesting that this state may simply be two glueballs. Given the significant discretization errors in the single glueball scaling data, one would expect similar systematic errors in the two glueball state. The absence of any level of similar mass in all other channels justifies the spin-zero interpretation of this state. Considering the difficulties encountered in extrapolating the lowest-lying scalar to the continuum limit, we made no serious attempt to determine the continuum limit of this first-excited state. However, the result of a fit using $\varphi_{2,4}$ is included in Table XIX. The possible interpretation of this level as a two glueball system might be strengthened by a more precise finitevolume study.

\section{Conversion to physical units}

In order to convert our glueball mass computations into physical units, we must specify the value of the hadronic scale. The hadronic scale $r_{0}$ has a precise definition in terms of the static-quark potential. However, the static-quark potential cannot be directly measured in an experiment; it must be deduced indirectly from other observables. We decided to use a variety of different physical quantities to deduce $r_{0}$.

In Table XX, estimates of $r_{0}^{-1}$ using the results from various quenched lattice simulations are shown. For each computation, the quantity used to set the lattice spacing, such as the mass of the $\rho$ or the $1 P-1 S$ splitting in heavy quarkonia, is indicated. The determination of $r_{0}^{-1}$ from $a^{-1}$ was accomplished using values of $a / r_{0}$ given in Ref. [16] for the Wilson gluonic action at various values of $\beta$, interpolating where necessary. Note that due to quenching effects, $r_{0}$ varies with the quantity used to set the scale. The entries in the last column of Table XX cannot be considered as different measurements of a single quantity and thus, strictly speaking, their weighted average has no statistical meaning. The last column of the table is meant to illustrate the range of values one obtains for $r_{0}^{-1}$ when using various scale setting quantities. We expect that the value of $r_{0}^{-1}$ appropriate for the low-lying glueballs should lie somewhere within this range. The simple average $r_{0}^{-1}=410 \pm 20 \mathrm{MeV}$ of the determinations in Table XX was taken as our estimate of the hadronic scale.

For our final continuum mass estimates of the tensor glueball, we found $2400 \pm 10 \pm 120 \mathrm{MeV}$ (where the first error is

TABLE XIX. Extrapolations of the scalar glueball mass estimates to the continuum limit. The fit functions used are given in Eqs. (20) and (21). The value indicated in bold is our final continuum mass estimate for the scalar glueball.

\begin{tabular}{lcccccc}
\hline \hline State & Fit function & $r_{0} m_{G}$ & \multicolumn{1}{c}{$c_{2}$} & $c_{4}$ & $c_{L}$ & $\chi^{2} / N_{\mathrm{DF}}$ \\
\hline$A_{1}^{++}$ & $\varphi_{1 L}$ & $\mathbf{3 . 9 8 ( 1 5 )}$ & $-18(4)$ & $18(5)$ & $0.96(13)$ & 0.25 \\
& $\varphi_{2,4}$ & $3.86(8)$ & $-3.5(4)$ & $4.5(5)$ & & 0.55 \\
$A_{1}^{*++}$ & $\varphi_{2,4}$ & $6.93(19)$ & $-5.3(1.1)$ & $8.8(1.4)$ & & 0.41 \\
\hline \hline
\end{tabular}


TABLE XX. Estimates of $r_{0}^{-1}$ using results from various quenched lattice simulations with the Wilson gluonic action. The simple average $r_{0}^{-1}=410(20) \mathrm{MeV}$ of the last column is taken as our estimate.

\begin{tabular}{|c|c|c|c|c|c|}
\hline Source & $\beta$ & Quark action & Scale setting quantity & $a^{-1}(\mathrm{GeV})$ & $r_{0}^{-1}(\mathrm{MeV})$ \\
\hline NRQCD [23] & 6.0 & NRQCD & $\Upsilon(2 S-1 S, 1 P-1 S)$ & $2.4(1)$ & $434(23)$ \\
\hline NRQCD [24] & 5.7 & NRQCD & $J / \psi(1 P-1 S)$ & $1.23(4)$ & $430(16)$ \\
\hline LANL [25] & 6.0 & Wilson & $M_{\rho}$ & $2.330(41)$ & $422(16)$ \\
\hline GF11 [26] & 6.17 & Wilson & $M_{\phi}$ & $2.93(11)$ & $419(17)$ \\
\hline JLQCD [27] & 6.1 & Wilson & $J / \psi(1 P-1 S)$ & $2.54(7)$ & $394(13)$ \\
\hline JLQCD [27] & 6.3 & Wilson & $J / \psi(1 P-1 S)$ & $3.36(11)$ & 401(14) \\
\hline JLQCD [28] & 6.3 & Wilson & $M_{\rho}$ & $3.41(20)$ & $406(24)$ \\
\hline Fermilab [29] & 6.1 & Fermilab & $J / \psi(1 P-1 S)$ & $2.55(8)$ & $395(14)$ \\
\hline BLS [30] & 6.3 & Heavy light & $f_{\pi}$ & $3.21(9)$ & $383(11)$ \\
\hline BLS [30] & 6.3 & Heavy light & $M_{\rho}$ & $3.44(9)$ & $410(11)$ \\
\hline UKQCD [31] & 6.2 & Wilson & $M_{\rho}$ & $2.77(16)$ & $376(22)$ \\
\hline
\end{tabular}

statistical and the second is from uncertainties in the determination of $\left.r_{0}\right)$. It is interesting to note that our mass estimate lies within $8 \%$ of the mass of the $f_{J}(2220)$ resonance $[17,18]$, reported to have quantum numbers (even) ${ }^{++}$. In order to make a direct comparison with experiment, however, corrections to our result from light quark effects and mixings with nearby conventional mesons must be taken into account. Our estimate of the mass of the first-excited glueball in the tensor channel was $3320 \pm 20 \pm 160 \mathrm{MeV}$; for the pseudovector state, we found a mass of 2960土 $10 \pm 140 \mathrm{MeV}$. Our estimate from the fit using $\varphi_{1 L}$ for the mass of the scalar glueball was $1630 \pm 60 \pm 80 \mathrm{MeV}$; however, we regard the continuum limit extrapolation for this state as being less reliable than those for the other glueballs.

\section{E. Comparison of efficiencies}

A quantitative comparison of efficiencies is difficult to make. There are many factors which affect the overall efficiency of a Monte Carlo simulation. Certainly, the number of link updates is an important factor. The speed of an update is, of course, platform, action, and algorithm dependent. On the DEC Alpha workstations we used, a CM update using an improved action required twice as much time as for the Wilson action; the improved-action OR updating time was three times longer. Critical slowing down and thermalization are also contributing factors, but a crucial issue is the reduction of excited-state contaminations in the glueball correlators. In our coarse lattice simulations, we found that current methods for constructing good glueball operators were very effective in hastening the onset of plateaus in the effective masses.

Given these difficulties in assessing the efficiency of a glueball simulation, we decided to make our comparisons based simply on the number of link updates $N_{l u}$ and the fractional error $\epsilon$ attained in the final mass estimates. Since the error in a Monte Carlo estimate decreases with the number of measurements $N$ as $1 / \sqrt{N}$, we expect that the reciprocal product of the number of link updates and the square of the fractional error is approximately proportional to the efficiency of a simulation; we denote this quantity by $\mathcal{E}=1 /\left(\epsilon^{2} N_{l u}\right)$. An interesting comparison to make is between simulations at a small lattice spacing, such as $a_{s} \sim 0.05 \mathrm{fm}$, using the Wilson action and improved-action simulations at a spacing $a_{s} \sim 0.2 \mathrm{fm}$. Such a comparison is relevant because the discretization errors in these cases are of comparable magnitude, excepting the scalar glueball mass. For the $\beta=6.4$ Wilson action run in Ref. [19] by the GF11 Collaboration using gauge-invariant glueball operators, a total of $3.13 \times 10^{12}$ link updates were performed, an error of $2.5 \%$ was achieved in the scalar glueball mass, and a fractional error of $3.6 \%$ was obtained for the tensor mass. Using $r_{0}=0.48 \mathrm{fm}$ and $a_{s} / r_{0}=0.101(2)$, the lattice volume in this simulation was $(1.55 \mathrm{fm})^{2} \times(1.45 \mathrm{fm})$. For the same value of $\beta$ in Ref. [20] by the UKQCD Collaboration, $1.35 \times 10^{11}$ link updates were made and fractional errors $3.4 \%, 3.3 \%$, and $9 \%$ were obtained for the scalar, tensor, and pseudovector masses, respectively. The lattice volume was $(1.55 \mathrm{fm})^{3}$ for this simulation. In our $\beta=2.6, \xi=3$ run, $5.76 \times 10^{9}$ link updates were performed and $1.5 \%, 1.0 \%$, and $1.0 \%$ errors in the scalar, tensor, and pseudovector masses were achieved. Our lattice volume was $(1.93 \mathrm{fm})^{3}$ since $a_{s} / r_{0}=0.4021(9)$. For our $\beta=2.4, \xi=5$ run, the number of link updates was $9.83 \times 10^{9}$ and the errors obtained in the scalar, tensor, and pseudovector masses were $1.0 \%, 0.5 \%$, and $0.8 \%$, respectively. Using $a_{s} / r_{0}=0.459$ (1), our lattice volume for this run was $(1.76 \mathrm{fm})^{3}$. Thus, the ratios of the $\mathcal{E}$ values for our $\beta=2.6$ and $\beta=2.4$ simulations to those of the GF11 run were 1500 and 2000, respectively, for the scalar glueball mass, and 7000 and 17000 for the tensor glueball mass. The ratios of the $\beta=2.6$ and $\beta=2.4 \mathcal{E}$ values to those of the UKQCD run were 120 and 160, respectively, for the scalar mass, 260 and 600 for the tensor, and 1900 and 1700 for the pseudovector.

Considering our ten simulations together, a total of $5 \times 10^{10}$ link updates were performed. For the Wilson action simulations of Refs. [19,20], an estimated $10^{13}$ and $10^{12}$ link updates were required, respectively, to generate continuum limit results whose statistical uncertainties were larger (for the tensor and pseudovector states) than those quoted here: the statistical error on our estimate for $r_{0} m\left(2^{++}\right)$was about five times smaller than that from the extrapolation of the Wilson action results, and for $r_{0} m\left(1^{+-}\right)$, the uncertainty was 20 times smaller, implying that about 25-400 times greater statistics would be required for similar accuracy. Thus, in total, the anisotropic lattice simulations were certainly more than 1000 times more efficient. 
The above discussion illustrates the computational advantages of extracting nonscalar glueball masses from simulations on coarse, anisotropic lattices $\left(a_{s} \sim 0.2 \mathrm{fm}\right)$ using an improved action instead of lattices for which $a_{s} \sim 0.05 \mathrm{fm}$. The excellent overlaps achieved from our variational calculations demonstrate another advantage of simulating on coarse spatial lattices. The glueball wave functions extend over only a few points of the lattice when $a_{s} \approx 0.2 \mathrm{fm}$. Thus, variational calculations using a feasible number of basis functions (a dozen or so) can yield very good approximations to the glueball wave functions. This will be especially important for future decay constant calculations and determinations of mixings with nonglueball states. These advantages have already enabled us to study the more massive glueball states which have yet to be simulated reliably using the Wilson action; these results will be reported elsewhere.

These advantages are less clear for the scalar glueball mass due to the presence of $20 \%$ discretization errors at $a_{s} \sim 0.2 \mathrm{fm}$. Using the action of Eq. (1), simulations at one or more lattice spacings smaller than $0.2 \mathrm{fm}$ would be needed to firmly establish the continuum limit. A more attractive approach would be to use an action for which discretization errors in the scalar glueball mass at $a_{s} \sim 0.2 \mathrm{fm}$ are negligibly small. The search for such an action is currently underway.

\section{CONCLUSION}

We have demonstrated the advantages of using anisotropic lattices and an improved gluonic action for simulating glueballs. Ten simulations at lattice spacings ranging from 0.2 to $0.4 \mathrm{fm}$ were performed using DEC Alpha workstations, and the results were extrapolated to the continuum limit. Results for the masses of the scalar $\left(0^{++}\right)$, the tensor $\left(2^{++}\right)$, and the pseudovector $\left(1^{+-}\right)$glueballs in $\mathrm{SU}(3)$ pure-gauge theory were presented in terms of the hadronic scale $r_{0}^{-1}$. The continuum limits for the tensor and pseudovector glueball masses were obtained with uncertainties of less than $1 \%$, significantly improving upon previous estimates from Wilson action simulations carried out with the aid of supercomputer resources. Extrapolation of the scalar glueball mass to the continuum limit was hampered by uncertainties in choosing the fitting function and discretization errors which were 20-25\% even for our smallest lattice spacings; although uncomfortably large, these finite-spacing errors were half as large as those obtained using the Wilson action. Finite-volume errors in our results were shown to be negligible. The masses of the first-excited states in the scalar and tensor channels were also examined.

Our results show that spatially coarse, anisotropic lattice simulations are an effective means of studying gluonic systems. The techniques exploited here are sufficiently powerful to overcome the difficulties which plague Monte Carlo calculations involving gluonic excitations. These methods should be useful for studying the spectrum of heavier glueball states. Data for the masses of all 20 lattice irreps of the cubic group (including parity and charge conjugation) are currently being accumulated in order to survey the spectrum of SU(3) glueball states below $4 \mathrm{GeV}$ comprehensively. We shall report on these results in the near future. We also plan to use the techniques outlined in this paper to determine various glueball matrix elements and decay strengths, to investigate the mixings of glueballs with conventional hadronic states, and to study mesonic states containing excited glue (the so-called hybrid mesons). The size of the discretization errors in the scalar glueball mass was the only disappointing aspect of this work; we are currently investigating a new class of lattice actions with the hope of reducing these lattice artifacts for $a_{s} \sim 0.2 \mathrm{fm}$ to the level of a few percent.

\section{ACKNOWLEDGMENTS}

We would like to thank Peter Lepage, Julius Kuti, Chris Michael, Terrence Draper, and Keh-Fei Liu for helpful discussions. This work was supported by the U.S. DOE, Grant No. DE-FG03-90ER40546. M.P. is grateful to the University of Kentucky Center for Computational Sciences for financial support.
[1] C. Morningstar and M. Peardon, in Lattice '95, Proceedings of the International Symposium, Melbourne, Australia, edited by T. D. Kieu et al. [ Nucl. Phys. B (Proc. Suppl.) B47, 258 (1996)].

[2] C. Morningstar, in Lattice '96, Proceedings of the International Symposium, St. Louis, Missouri, edited by C. Bernard et al. [Nucl. Phys. B (Proc. Suppl.) B53, 914 (1997)].

[3] C. Morningstar and M. Peardon, in Lattice '96 [2], p. 917.

[4] M. Alford et al. (in preparation).

[5] F. Brandstaeter, A. Kronfeld, and G. Schierholz, Nucl. Phys. B345, 709 (1990).

[6] M. Albanese et al., Phys. Lett. B 192, 163 (1987).

[7] M. Teper, Phys. Lett. B 183, 345 (1986); K. Ishikawa, A. Sato, G. Schierholz, and M. Teper, Z. Phys. C 21, 167 (1983).

[8] Y. Liang et al., Phys. Lett. B 307, 375 (1993).

[9] B. Berg and A. Billoire, Nucl. Phys. B221, 109 (1983).

[10] R. Sommer, Nucl. Phys. B411, 839 (1994).

[11] G. Parisi et al., Phys. Lett. 128B, 418 (1983).
[12] G. Schierholz, in Lattice '88, Proceedings of the International Symposium, Batavia, Illinois, edited by A. S. Kronfeld and P. B. Mackenzie [Nucl. Phys. B (Proc. Suppl.) B9, 244 (1989)].

[13] M. Lüscher, Commun. Math. Phys. 104, 177 (1986).

[14] U. Heller, Phys. Lett. B 362, 123 (1995).

[15] A. Patel et al., Phys. Rev. Lett. 57, 1288 (1986).

[16] G. S. Bali and K. S. Schilling, Int. J. Mod. Phys. C 4, 1167 (1993); and (private communication); H. Wittig, in Lattice '94, Proceedings of the International Symposium, Bielefeld, Germany, edited by F. Karsch et al. [ Nucl. Phys. B (Proc. Suppl.) B42, 288 (1995)].

[17] Particle Data Group, R. M. Barnett et al., Phys. Rev. D 54, 1 (1996).

[18] R. M. Baltrusaitis et al., Phys. Rev. Lett. 56, 107 (1986).

[19] H. Chen et al., in Lattice '93, Proceedings of the International Symposium, Dallas, Texas, edited by T. Draper et al. [ Nucl. Phys. B (Proc. Suppl.) B34, 357 (1994)].

[20] G. S. Bali et al., Phys. Lett. B 309, 378 (1993). 
[21] C. Michael and M. Teper, Nucl. Phys. B314, 347 (1989).

[22] P. de Forcrand et al., Phys. Lett. 160B, 137 (1985).

[23] C. Davies et al., Phys. Rev. D 50, 6963 (1994).

[24] C. Davies et al., Phys. Rev. D 52, 6519 (1995).

[25] T. Bhattacharya et al., Phys. Rev. D 53, 6486 (1996).

[26] F. Butler et al., Nucl. Phys. B430, 179 (1994).
[27] S. Aoki et al., in Lattice '96 [2], p. 355.

[28] S. Aoki et al., in Lattice '96 [2], p. 349.

[29] A. X. El-Khadra, in Lattice '93 [19], p. 141.

[30] C. Bernard, J. Labrenz, and A. Soni, Phys. Rev. D 49, 2536 (1994).

[31] C. R. Allton et al., Phys. Lett. B 284, 377 (1992). 